NASA Technical Memorandum 85932

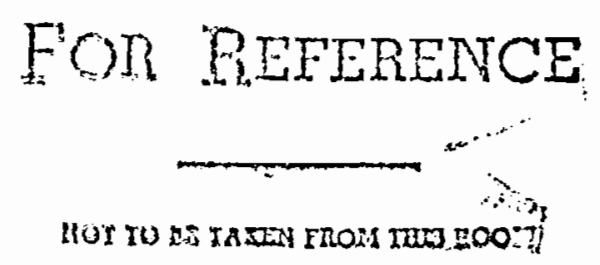

\title{
Numerical Simulation of the Tip Vortex Off a Low-Aspect-Ratio Wing at Transonic Speed
}

N.N. Mansour

April 1984

\section{LERRAEY GOPY \\ 1691984}

LANGLEY RESEARCH CENTER

LIGRARY, NASA

IIAUETON, VIRGINIA

\section{N/SN}





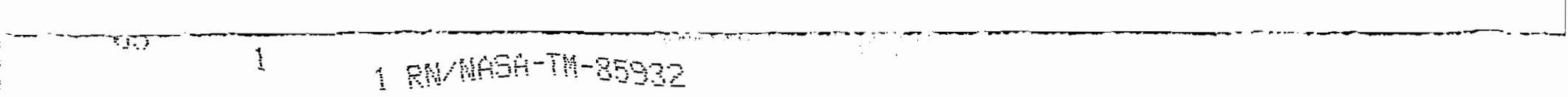


$\mathrm{A}^{2}$

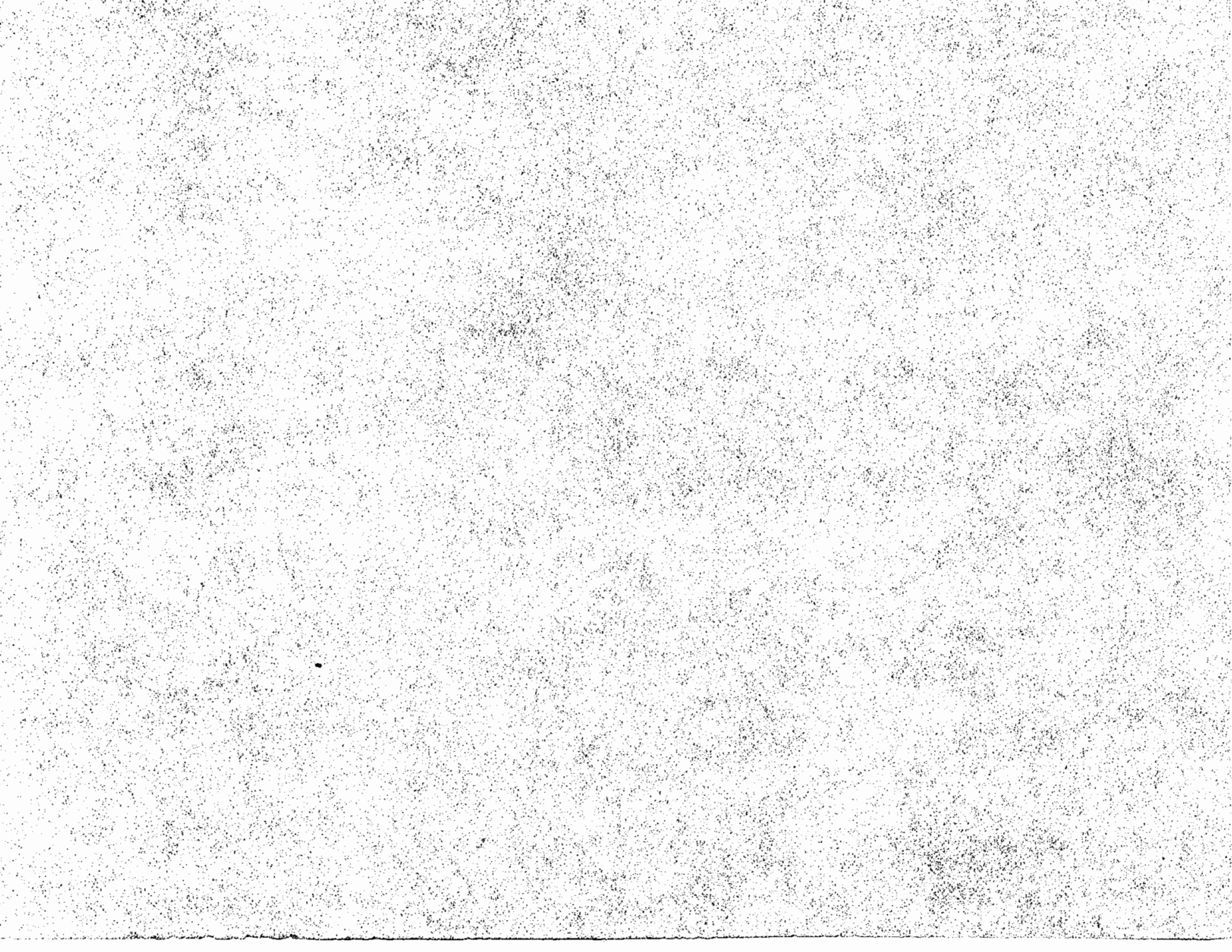


NASA Technical Memorandum 85932

\section{Numerical Simulation of the Tip Vortex Off a Low-Aspect-Ratio Wing at Transonic Speed}

N. N. Mansour, Stanford University

Prepared for Thermo- and Gas-Dynamics Division

Ames Research Center

\section{N/Sก}

National Aeronautics and

Space Administration

Ames Research Center

Moffett Field, California 94035 
is

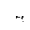

- 


\title{
NUMERICAL SIMULATION OF THE TIP VORTEX OFF A LOW-ASPECT-RATIO WING AT TRANSONIC SPEED
}

\author{
N. N. Mansour* \\ Stanford University, Stanford, California
}

\begin{abstract}
The viscous transonic flow around a low-aspect-ratio wing has been computed using an implicit, three-dimensional, "thin-layer" Navier-Stokes solver. The grid around the geometry of interest is obtained numerically as a solution to a Dirichlet problem for the cube. The geometry chosen for this study is a low-aspect-ratio wing with large sweep, twist, taper, and camber. The topology chosen to wrap the mesh around the wing with good tip resolution is a C-O type mesh. Using this grid, the flow around the wing was computed for a free-stream Mach number of 0.82 at an angle of attack of $5^{\circ}$. At this Mach number, an oblique shock forms on the upper surface of the wing, and a tip vortex and three-dimensional flow separation off the wing surface are observed. Particle path lines indicate that the three-dimensional flow separation on the wing surface is part of the roots of the tip-vortex formation. The llrung or the tip vortex berore the wing tralling eage is clearly observed by following the trajectory of particles released around the wing tip.
\end{abstract}

\section{Introduction}

It is well known from classic wing theory ${ }^{1}$ that the presence of a vortex wake is a necessary condition for all finite-span lifting wings. The most advanced fullpotential numerical methods for the simulation of subsonic and transonic flows combine classic wing theory (vortex sheet trailing the wing at a fixed spatial location) with the full-potential calculation to compute realistic lift values for wings of fairly general shapes. ${ }^{2}$ In reality, this vortex sheet will roll up into discrete vortices. ${ }^{1}$ This roll up starts close to the tip of the wing where the flow is predominantly threedimensional and possesses a concentrated (rolled-up) vortex structure. The wake vortex generated by large transport aircraft is known for its potential hazard to smaller aircraft. This hazard is the major impediment to increasing traffic capacity at major air terminals and a large effort is being directed to minimize it. ${ }^{3}$ In the

\footnotetext{
*Present address: Sandia National Laboratories, Livermore, Calif. 94550.
} 
case of low-aspect-ratio wings, the tip vortex contributes directly to lift and drag and, hence, it is important in practical applications.

To contribute to our understanding of the formation of the tip vortex, a mathematical model is needed in which the appearance of the tip vortex is a result of the solution rather than an empirical input to the solution. At high Reynolds number, the simplest model seems to be the thin-layer approximation ${ }^{4}$ to the Navier-Stokes equations, since the vortex formation is a viscous phenomenon generated in a thin layer close to the surface of the wing, whereas most of the flow field is convection dominated. The model that incorporates the full viscous terms is preferable when a fine mesh is used in all three-directions. However, experience with two-dimensional computations indicates that the difference between the results from a full Navier-Stokes calculation and a thin-layer calculation is negligible when a coarse grid is used in the streamwise direction. The objective of this paper is to study the formation of the tip vortex off a low-aspect-ratio wing. The thin-layer approximation is used as a first model for this purpose. Vortex formation and capturing at wing tips are reported by Rizzi and Eriksson ${ }^{5}$ and Eriksson and Rizzi ${ }^{6}$ who use the Euler equations as the mathematical model. It is not clear why a tip vortex is observed in inviscid (Euler) flow computations, and the understanding of this observation is still under investigation by Rizzi. ${ }^{7}$

Considerable progress has been made in developing the solution methods for the nonlinear equations of fluid motion. For a review of the achievements of the numerical methods in computing transonic flows, the reader is referred to the recent article of Mehta and Lomax. ${ }^{8}$ The methods range from the fully explicit to the factored fully implicit schemes. The application of these methods to three-dimensional flows has been restricted mostly to simple geometries. Both the computational efforts involved and the problem of grid generation over complicated geometries seem to hinder the application of the available computational methods to three dimensions.

The procedure followed in this work is to treat the mesh-generation problem and the solution to the governing equations as two separate operations. This appears to be the approach followed by most authors ${ }^{6, \theta, 10}$ to compute the three-dimensional flow over complicated geometries. The adaptive gridding approach ${ }^{11}$ is still under development, and its application to three-dimensions for complicated geometries is still not proved. The mesh-generation method chosen to generate the grid around the geometry of interest is the elliptic mesh generator described in Sec. II. The geometry chosen for this study is a low-aspect-ratio $\left(A_{r}=0.85\right)$ wing with $45^{\circ}$ sweep, $8.3^{\circ}$ twist, and 0.3 taper. The mathematical model used to compute the flow around this wing is the thin-layer Navier-Stokes equations in generalized coordinates (described in Sec. III). The numerical method used to solve these equations is the factored implicit scheme $\mathrm{e}^{12,13}$ as applied to three-dimensional aerodynamics 
applications by Pulliam and Steger. ${ }^{14}$ The data-management strategy described in Sec. IV allows the use of fairly fine three-dimensional meshs (up to 200,000 grid nodes). The computed results (using 75,000 grid points) for the formation of the tip vortex over a low-aspect-ratio wing at a transonic Mach number $(M=0.82)$ are presented in Sec. V.

\section{Grid Generation}

The grid around the geometry of interest is obtained numerically as a solution to a Dirichlet problem for the cube. The approach relies on the method of Mastin and Thompson, ${ }^{15}$ with the source terms specified using a variation of the method of Thomas. ${ }^{16}$ The source terms in this approach are specified so that the Poisson equations possess exponential solutions for locally constant parameters. This allows grid control inside the volume by the grid-point distribution assigned on the surface of the domain. The distribution on the surface of the domain is controlled by the grid-point distribution along the boundary of the surface using the method of Thomas and Middlecoff. ${ }^{17}$

\section{Generation Procedure}

The first task is to distribute the grid points on the boundary of the six surfaces of the volume of interest. This is done using a composite transformation consisting of a hyperbolic-tangent and a hyperbolic-sine functions as follows (T. Morel, private communication, Integral Technologies, Westmont, 1ll., 1983.):

$$
\begin{aligned}
a\left(x-x_{0}\right) & =\sinh (c \Gamma+d) \\
e \xi+f & =\tanh ^{-1}(g \Gamma+h)
\end{aligned}
$$

where $x$ is the physical coordinate $\epsilon\left[x_{1}, x_{2}\right], \xi$ is the transformed coordinate $\epsilon$ $[1, N]$, and $\Gamma$ is an intermediate coordinate $\epsilon[1, N] ; N$ is the total number of grid points. To determine the seven constants $a, c, d, e, f, g$, and $h$, the following four conditions are imposed:

$$
\left.\left.\begin{array}{l}
\xi=1 \\
\Gamma=1
\end{array}\right\} \text { when } x=x_{1}, \quad \text { and } \begin{array}{l}
\xi=N \\
\Gamma=N
\end{array}\right\} \text { when } x=x_{2}
$$

We are left with three adjustable stretching parameters that we specify as follows:

$$
\begin{aligned}
& t_{1}=g+h \\
& t_{2}=g N+h \\
& t_{3}=c N+d
\end{aligned}
$$


Parameters $t_{1}$ and $t_{2}$ provide grid clustering around $x_{1}$ and $x_{2}$, and $t_{3}$ provides grid clustering around $x_{0}$.

Once the distribution of the grid points on the boundary of the surface has been determined, a two-dimensional elliptic grid generator is used to distribute the grid point on the surface. The generating system is of the form

$$
\begin{array}{r}
\alpha\left(x_{\xi \xi}+\phi x_{\xi}\right)-2 \beta x_{\xi \eta}+\gamma\left(x_{\eta \eta}+\psi x_{\eta}\right)=0 \\
\alpha\left(y_{\xi \xi}+\phi y_{\xi}\right)-2 \beta y_{\xi \eta}+\gamma\left(y_{\eta \eta}+\psi y_{\eta}\right)=0
\end{array}
$$

where

$$
\begin{aligned}
& \alpha=x_{\eta}^{2}+y_{\eta}^{2} \\
& \beta=x_{\xi} x_{\eta}+y_{\xi} y_{\eta} \\
& \gamma=x_{\xi}^{2}+y_{\xi}^{2}
\end{aligned}
$$

Imposing the orthogonality condition at the boundary on the grid lines yields the values of $\phi$ and $\psi$ on the boundary as follows: ${ }^{17}$

$$
\begin{aligned}
& \phi=-\left(x_{\xi} x_{\xi \xi}+y_{\xi} y_{\xi \xi}\right) /\left(x_{\xi}^{2}+y_{\xi}^{2}\right) \text { on } \eta=1, N_{\eta} \\
& \psi=-\left(x_{\eta} x_{\eta \eta}+y_{\eta} y_{\eta \eta}\right) /\left(x_{\eta}^{2}+y_{\eta}^{2}\right) \text { on } \xi=1, N_{\xi}
\end{aligned}
$$

The values of the parameter $\phi$ (or $\psi$ ) at the interior are then computed by linear interpolation along $\xi=$ const ( $\eta=$ const) lines.

In the above formulations, the boundary is assumed to be rectilinear and the surface is assumed to be planar. For general lines (or surfaces) the grid is generated on an appropriate rectilinear (or planar) projection, and the points on the line (or surface) are deduced from the points on the projection. A more general method that accounts for the effects of curvature would require specifying the tangents and radii of curvature, which in general may not be known analytically.

Once the grid is generated on the six surfaces of the volume, the mapping between the physical space $(x, y, z)$ and the computational space $(\xi, \eta, \zeta)$ is determined by solving the following set of transformed Poisson equations:

$$
\begin{array}{r}
\alpha_{1}\left(x_{\xi \xi}+\phi x_{\xi}\right)+\alpha_{2}\left(x_{\eta \eta}+\psi x_{\eta}\right)+\alpha_{3}\left(x_{s \varsigma}+\omega x_{\varsigma}\right)+ \\
2\left(\beta_{1} x_{\xi \eta}+\beta_{2} x_{\eta \xi}+\beta_{3} x_{\varsigma \xi}\right)=0 \\
\alpha_{1}\left(y_{\xi \xi}+\phi y_{\xi}\right)+\alpha_{2}\left(y_{\eta \eta}+\psi y_{\eta}\right)+\alpha_{3}\left(y_{s \zeta}+\omega y_{\xi}\right)+ \\
2\left(\beta_{1} y_{\xi \eta}+\beta_{2} y_{\eta \xi}+\beta_{3} y_{s \xi}\right)=0 \\
\alpha_{1}\left(z_{\xi \xi}+\phi z_{\xi}\right)+\alpha_{2}\left(z_{\eta \eta}+\psi z_{\eta}\right)+\alpha_{3}\left(z_{s \zeta}+\omega z_{\xi}\right)+ \\
2\left(\beta_{1} z_{\xi \eta}+\beta_{2} z_{\eta \zeta}+\beta_{3} z_{\varsigma \xi}\right)=0
\end{array}
$$


where

$$
\begin{aligned}
& \alpha_{1}=\xi_{x}^{2}+\xi_{y}^{2}+\xi_{z}^{2} \\
& \alpha_{2}=\eta_{x}^{2}+\eta_{y}^{2}+\eta_{z}^{2} \\
& \alpha_{3}=\zeta_{x}^{2}+\zeta_{y}^{2}+\zeta_{z}^{2} \\
& \beta_{1}=\xi_{x} \eta_{x}+\xi_{y} \eta_{y}+\xi_{z} \eta_{z} \\
& \beta_{2}=\eta_{x} \zeta_{x}+\eta_{y} \zeta_{y}+\eta_{z} \zeta_{z} \\
& \beta_{3}=\zeta_{x} \xi_{x}+\zeta_{y} \xi_{y}+\zeta_{z} \xi_{z}
\end{aligned}
$$

The metric terms $\xi_{x}, \xi_{y}, \ldots$ are expressed in terms of $x_{\xi}, x_{\eta}, \ldots$ as follows:

$$
\begin{aligned}
& \xi_{x}=J\left(y_{\eta} z_{\varsigma}-y_{\varsigma} z_{\eta}\right) \quad \eta_{x}=J\left(z_{\xi} y_{\varsigma}-z_{\varsigma} y_{\xi}\right) \\
& \xi_{y}=J\left(z_{\eta} x_{\varsigma}-z_{s} x_{\eta}\right) \quad \eta_{y}=J\left(x_{\xi} z_{\zeta}-x_{\varsigma} z_{\xi}\right) \\
& \xi_{z}=J\left(x_{\eta} y_{\varsigma}-x_{\varsigma} y_{\eta}\right) \quad \eta_{z}=J\left(y_{\xi} x_{\varsigma}-y_{\varsigma} x_{\xi}\right) \\
& \zeta_{x}=J\left(y_{\xi} z_{\eta}-y_{\eta} z_{\xi}\right) \\
& \zeta_{y}=J\left(z_{\xi} x_{\eta}-z_{\eta} x_{\xi}\right) \\
& \zeta_{z}=J\left(x_{\xi} y_{\eta}-x_{\eta} y_{\xi}\right)
\end{aligned}
$$

and

$$
\begin{aligned}
J^{-1}= & x_{\xi} y_{\eta} z_{\varsigma}+x_{\varsigma} y_{\xi} z_{\eta}+x_{\eta} y_{\varsigma} z_{\xi} \\
& -x_{\xi} y_{\varsigma} z_{\eta}-x_{\varsigma} y_{\eta} z_{\xi}-x_{\eta} y_{\xi} z_{\varsigma}
\end{aligned}
$$

Direct extension of the two-dimensional formulation [Eq. (4)] to three dimensions yields the values of $\phi, \psi$, and $\omega$ on the boundary as follows:

$$
\begin{aligned}
& \phi \quad=-\left(x_{\xi} x_{\xi \xi}+y_{\xi} y_{\xi \xi}+z_{\xi}^{z} z_{\xi \xi}\right) /\left(x_{\xi}^{2}+y_{\xi}^{2}+z_{\xi}^{2}\right) \\
& \text { on } \eta=1, N_{\eta} \text { and } s=1, N_{\mathrm{s}} \\
& \psi=-\left(x_{\eta} x_{\eta \eta}+y_{\eta} y_{\eta \eta}+z_{\eta} z_{\eta \eta}\right) /\left(x_{\eta}^{2}+y_{\eta}^{2}+z_{\eta}^{2}\right) \\
& \text { on } \xi=1, N_{\xi} \text { and } \zeta=1, N_{\zeta} \\
& \omega=-\left(x_{s} x_{s s}+y_{s} y_{s s}+z_{s} z_{s s}\right) /\left(x_{s}^{2}+y_{s}^{2}+z_{s}^{2}\right) \\
& \text { on } \xi=1, N_{\xi} \text { and } \eta=1, N_{\eta}
\end{aligned}
$$

The continuous representation of the parameters is found in planes $\xi=$ const, $\eta=$ const, and $\zeta=$ const by solving analytically the following equations:

$$
\begin{aligned}
& \phi_{\eta \eta \zeta S}=0 \\
& \psi_{\xi \xi \varsigma s}=0 \\
& \omega_{\xi \xi \eta \eta}=0
\end{aligned}
$$

The solutions to Eqs. (13) are elementary extensions to two-dimensions of the onedimensional linear interpolation method. 
The geometry chosen for this study is the low-aspect-ratio wing (wing C) investigated experimentally and numerically by Hinson and Burdges. ${ }^{18}$ The same geometry was also investigated experimentally by Keener (private communication, NASA Ames Research Center, Moffett Field, Calif., 1982.) and numerically by Holst et al. ${ }^{10}$ The characteristics of the wing are described in Fig. 1: 0.85 aspect ratio, $45^{\circ}$ sweep, $8.3^{\circ}$ twist, 0.3 taper.

In these numerical studies, the full-potential equation was used to compute the flow around the wing. With the full-potential methods, the vortex sheets are fitted rather than captured, and, therefore, the wrap around the wing tip is not critical. If the flow around the wing tip is of interest, then a mesh that wraps around the tip is desirable. Different grid wraps can be used to resolve the flow field around the wing that will achieve for a given total number of grid points concentration of grid points in different regions of the field. Rizzi and Eriksson ${ }^{5}$ used the density of grid points around the wing as a criterion by which to judge the different grid wraps and concluded that the $\mathrm{O}-\mathrm{O}$ and $\mathrm{C}-\mathrm{O}$ are the two "best" types. The $\mathrm{C}-\mathrm{O}$ type mesh shown in Fig. 2 was chosen in this study.

As pointed out by Rizzi and Eriksson, in order to map the wrap into a box-like region we need to introduce artificial singular lines. The location of these lines should be away from areas of interest since the flow field in the neighborhood of these lines may be in doubt. The map into the computational box chosen for this study is shown in Fig. 3. The cuts were chosen to be on the leading edge and trailing edge leaving the area around the tip free from these cuts. The wing and wake surface is mapped into the "bottom" surface of the parametric box (plane $\zeta=1$ ); the outer boundary (except the downstream surface) is mapped into the "top" surface (plane $\zeta=N_{\varsigma}$ ); the upper plane of symmetry is mapped into the "front" surface of the box (plane $\eta=1$ ); the lower plane of symmetry is mapped into the "back" surface of the box (plane $\eta=N_{\eta}$ ); the cut at the leading edge is mapped into the "left" surface of the box (plane $\xi=1$ ); and the downstream surface of the outer boundary plane is mapped into the "right" surface of the box (plane $\xi=N_{\xi}$ ).

\section{Governing Equations}

At high Reynolds numbers, the flow around the wing can be considered to be mostly inviscid, except in a thin-layer region close to the body where the viscous effects become important. With this in mind, the thin-layer approximation is used to simplify the Navier-Stokes equations in generalized coordinates to yield: ${ }^{14}$ 


$$
\partial_{\tau} q+\partial_{\xi} E+\partial_{\eta} F+\partial_{s} G=R e^{-1} \partial_{s} S
$$

where

$$
\begin{aligned}
& q=J^{-1}\left[\begin{array}{c}
\rho \\
\rho u \\
\rho v \\
\rho w \\
e
\end{array}\right], \quad E=J^{-1}\left[\begin{array}{c}
\rho U \\
\rho u U+\xi_{x} p \\
\rho v U+\xi_{y} p \\
\rho w U+\xi_{z} p \\
(e+p) U
\end{array}\right] \\
& F=J^{-1}\left[\begin{array}{c}
\rho V \\
\rho u V+\eta_{x} p \\
\rho v V+\eta_{y} p \\
\rho w V+\eta_{z} p \\
(e+p) V
\end{array}\right], \quad G=J^{-1}\left[\begin{array}{c}
\rho W \\
\rho u W+\varsigma_{x} p \\
\rho v W+\zeta_{y} p \\
\rho w W+\zeta_{z} p \\
(e+p) W
\end{array}\right] \\
& S=J^{-1} \times
\end{aligned}
$$

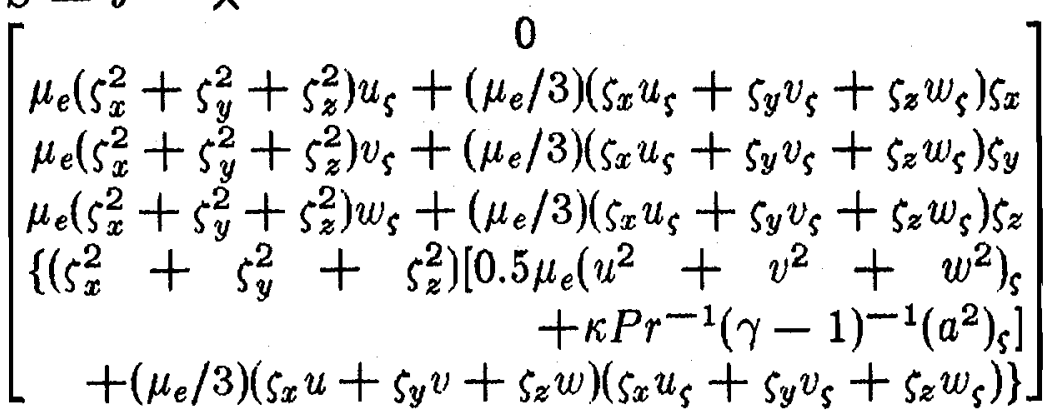

and

$$
\begin{aligned}
& U=\xi_{x} u+\xi_{y} v+\xi_{z} w \\
& V=\eta_{x} u+\eta_{y} v+\eta_{z} w \\
& W=\zeta_{x} u+\zeta_{y} v+\zeta_{z} w
\end{aligned}
$$

where $\mathrm{U}, \mathrm{V}$, and $\mathrm{W}$ are contravariant velocities written without metric normalization. The above set of equations is nondimensionalized using $a_{\infty}$ (the free-stream sound speed) for velocity, the cord length $(c)$ at the root of the wing for distances, and $\rho_{\infty}$ the free-stream density for density. One more equation of state is needed to close the above system, and we use the perfect gas law to relate the pressure, density, and velocity as follows:

$$
p=(\gamma-1)\left[e-0.5 \rho\left(u^{2}+v^{2}+w^{2}\right)\right]
$$

The effective viscosity in Eq. (16) is set equal to

$$
\mu_{e}=1+\mu_{t}
$$

where $\mu_{t}$ is a turbulent viscosity computed using the two-layer algebraic viscosity model of Baldwin and Lomax. ${ }^{4}$ 


\section{Numerical Method}

The approach followed in this work is the same as the one developed by Pulliam and Steger. ${ }^{14}$ The numerical algorithm is the implicit noniterative approximate factorization scheme of Beam and Warming. ${ }^{12}$ Fourth-order dissipation terms are added to the equations to control nonlinear instabilities, and implicit second difference terms operating on $\Delta q$ are added to extend the linear stability bound of the fourth-order terms.

\section{Linearization and Approximate-Factorization}

The equations of motion are differenced using a standard (non-staggered) mesh, central differencing in space, and backward (fully implicit) differencing in time. The differenced equations are linearized in time, then factored using the Douglas and $\mathrm{Gunn}^{20}$ technique to reduce the three-dimensional problem to three one-dimensional problems at a given time-level. The differenced equations are written as follows:

$$
D_{\xi} D_{\eta} D_{\varsigma} \Delta q=H
$$

where

$$
\begin{aligned}
\Delta q & =q^{n+1}-q^{n} \\
D_{\xi} & =I+h \delta_{\xi}\left(\partial E^{n} / \partial q^{n}\right)-\epsilon_{I} J^{-1} \nabla_{\xi} \Delta_{\xi} J \\
D_{\eta} & =I+h \delta_{\eta}\left(\partial F^{n} / \partial q^{n}\right)-\epsilon_{I} J^{-1} \nabla_{\eta} \Delta_{\eta} J \\
D_{\zeta} & =I+h \delta_{\zeta}\left(\partial G^{n} / \partial q^{n}\right)-h R e^{-1} \delta_{\zeta}\left(\partial S^{n} / \partial q^{n}\right) \\
& \quad-\epsilon_{I} J^{-1} \nabla_{\zeta} \Delta_{\zeta} J \\
H & =-\Delta t\left(\delta_{\xi} E^{n}+\delta_{\eta} F^{n}+\delta_{\zeta} G^{n}-R e^{-1} \delta_{\zeta} S^{n}\right) \\
& -\epsilon_{E} J^{-1}\left[\left(\nabla_{\xi} \Delta_{\xi}\right)^{2}+\left(\nabla_{\eta} \Delta_{\eta}\right)^{2}+\left(\nabla_{\zeta} \Delta_{\zeta}\right)^{2}\right] J q^{n}
\end{aligned}
$$

The $\delta$ 's are central-difference operators, $\nabla$ and $\Delta$ are backward and forward difference operators, and $h=\Delta t$ is the time-step. For a detailed description of the Jacobian matrices $\partial E^{n} / \partial q^{n}, \partial F^{n} / \partial q^{n}, \partial G^{n} / \partial q^{n}$, and $\partial S^{n} / \partial q^{n}$ the interested reader is referred to the paper of Pulliam and Steger. ${ }^{14}$

$\underline{\text { Data Management }}$

Proper resolution of the flow field around the wing requires a large number of grid points. Chapman ${ }^{21}$ estimated the following requirements to resolve the threedimensional boundary layer on a wing:

$$
\begin{aligned}
& N_{\xi}=4.5 n_{\xi} R e_{c}^{0.2} \\
& N_{\eta}=4.5 A_{r} n_{\eta} R e_{c}^{0.2} \\
& N_{\varsigma}=n_{\varsigma}
\end{aligned}
$$

where $n_{\xi}, n_{\eta}$, and $n_{\xi}$ are the average number of grid points per length $\delta$ (the boundary-layer thickness) in the streamwise, spanwise, and normal direction, respectively; $A_{r}$ is the aspect ratio; and $R e_{c}$ is the Reynolds number based on the cord 
length. For $R e_{c}=1 . \times 10^{8}, A_{r}=0.85, n_{\xi}=0.4, n_{\eta}=0.4$, and $n_{s}=15$, we need $N_{\xi}=30, N_{\eta}=30$, and $N_{\varsigma}=15$ to resolve the boundary layer on the upper surface. (Chapman recommends using $n_{\xi}=1, n_{\eta}=0.5$, and $n_{\varsigma}=20$.) Adding 10 grid points in the $\xi$-direction to capture the wake, doubling the grid points in the $\eta$-direction to resolve the lower surface, and adding 15 grid points in the $\zeta$-direction for the outer inviscid field yields a required total number of grid points equal to 72,000 . We need 19 words of memory at each grid point to store the independent variables and the metrics; the required total number of words of memory is then $1.4 \times 10^{6}$. The Cray1-S machine available at Ames Research Center is nominally a $1 \times 10^{6}$ words-in-core-memory machine. We then need to use the disk to store the flow-field data. To carry out the computation, part of the data are transferred into the core memory of the machine. The data-management algorithm devised is a five-plane algorithm.

The flow field is stored on the solid-state disk (SSD) and the "in-core" working set is a slab of five planes. With this scheme, data are transferred to the core of the machine in $\xi-\eta$ planes. When the data are needed in the $\zeta$-direction in more than five planes (for example for matrix inversion), a sweep through the data is carried out. The sweep through the data occurs in the $\zeta$-direction only. For a matrix inversion in the 5 -direction, two data-sweeps are needed. The solution proceeds as follows: during the first data-sweep, two matrices are inverted in each $\xi-\eta$ plane. Also, forward elimination for the third matrix inversion is completed. During the second sweep, the inversion of the third matrix is finished and the solution is advanced. A mesh up to 200,000 grid points can be handled by this algorithm.

\section{Boundary Conditions}

The physical boundary conditions are the conditions at infinity and the no-slip conditions on the surface of the wing. However, additional boundary conditions are needed when the equations are discretized. In general, boundary conditions on the six surfaces of the computational box are required to close the system of differenced equations. The use of appropriate boundary conditions is dependent on the mesh wrap. The type of boundary conditions used with the $\mathrm{C}-\mathrm{O}$ wrap in our case is shown in Fig. 3. At $\xi=1$, "cut" conditions are imposed. The cut boundary condition used in the present scheme is that the values at the cut are given as the average value of the two neighboring points in the computational domain. At $\xi=$ $N_{\xi}$, exit boundary conditions are imposed where the values at the boundary are extrapolated from the interior of the domain along $\xi$-lines. At $\eta=1$ and $\eta=N_{\eta}$, symmetry boundary conditions are imposed. At $\zeta=1$, no-slip boundary conditions are imposed on the surface of the wing and cut conditions are imposed in the wake. At $\zeta=N_{\varsigma}$, inflow conditions are imposed where the velocity, density, and Mach 
number are specified.

\section{Results}

Using the grid described in Sec. II, the flow around the wing was computed for free-stream Mach number $M=0.82$ at an angle of attack $\alpha=5^{\circ}$. The design conditions $^{18}$ for this wing were $M=0.85$ and $\alpha=5^{\circ}$. The results presented in this section are, hence, for a Mach number slightly below the design Mach number, and the flow around the wing is expected to be smooth. (Smooth flow is loosely defined in this paper as a flow that does not exhibit a massive separation.)

The surface-pressure contours (Fig. 4) and the Mach contours (Fig. 5) on a surface parallel to the surface of the wing show the presence of an oblique shock between $y \simeq 0.4 c$ and $y \simeq 0.75 c$ at about 0.25 the local cord length from the leading edge. This shock is also detected by the $C_{\mathrm{p}}$ profiles of the experimental data. In the region of the shock, our mesh is relatively coarse, as can be seen in Fig. 6 where the open symbols represent the computed $C_{\mathrm{p}}$ results at the mesh locations. Comparison of the computed $C_{\mathrm{p}}$ profile with the experimental data shows that the suction peak is overpredicted. No Mach-number or angle-of-attack corrections (for the presence of the walls in the experiment) were attempted to match the experimental data. Using the full-potential-equation formulation, Subramanian et al. ${ }^{2}$ found that by correcting the Mach number and the angle of attack they can match the experimental data at the different spanwise locations. In general, the shape of the $C_{\mathrm{p}}$ profiles predicted by the present computation is similar to the experimental profiles at the various spanwise locations, but the suction peak is overpredicted.

The flow pattern over the surface of the wing was visualized experimentally by Keener using an oil-flow technique. It was found that for low Mach numbers ( $M=0.7)$ the flow is smooth over the wing, but at high Mach numbers $(M>0.85)$ a massive three-dimensional separation is observed on the upper surface of the wing. The pattern indicated by the oil flow for $M=0.82$ is sketched in Fig. 7 from a photograph. The major feature of the flow at this Mach number is the appearance of a ridge where the oil path lines change direction. The flow-fleld pattern from the numerical solution can be best visualized by plotting the path of particles whose motion is defined by the following equation:

$$
\frac{d \vec{x}}{d t}=\vec{u}
$$

The above equation can also be used as the definition of the streamlines. If we restrict the path of the particles to a surface, we will be defining the streamlines 
of the projection of the velocity vector on that surface. On the surface of the wing the velocity is zero because of the no-slip condition and, hence, particles on the wing surface do not move. At one mesh $(\varsigma=2)$ above the surface, the velocity vector is nearly parallel to the surface of the wing, and by plotting the particle path in that plane we will be observing the streamlines close to the surface, but not simulating the oil-flow pattern. In general, the oil film has a thickness, mass, and surface tension, and a complete simulation of the film is much more involved than massless particle tracing. Figure 8 shows the particle path pattern in the $\zeta=2$ plane (average distance to the wing $d_{2}=0.4 \times 10^{-3} \mathrm{c}$ ). The appearance of a ridge is clearly seen where the paths of the particles converge. Converging lines indicate flow separation, and diverging lines indicate flow reattachment. Both separation and reattachment can be detected in Fig 8 . If we compare the computed streamlines in that plane $(\zeta=2)$ with the experimental oil-film pattern, we note the pronounced curving of the streamlines as opposed to the rather straight, streaky pattern indicated by the oil film. The difference between the two patterns may be due to the fact that the oil film has a thickness and may be protruding into the boundary layer beyond the plane at which the numerical results are presented. The streamline pattern will show less curving as we move out of the boundary layer (see the pattern in Fig. 9 at an average distance from the surface of the wing $d_{8}=6 \times 10^{-3} \mathrm{c}$ ).

Figure 10 shows the location of the separation line and reattachment line relative to the wing outline. Particles that start between the reattachment line and the wing tip are convected toward the tip vortex; particles outside that region will not be trapped by the tip vortex. The tip vortex can be clearly visualized by the three-dimensional path of the particles shown in Fig. 11. These particles are generated near the leading edge of the wing at several spanwise locations. Particles that start at a spanwise location $y<0.4 c$ do not get entrapped in the tip vortex, but particles starting at $y>0.4 c$ are captured in the tip vortex. The threedimensional-flow separation on the wing surface forms part of the roots of the tip vortex. Figures 12 show the tip vortex as detected by particles released close to the tip of the wing. These figures show the projection of the tip vortex line on an $x-y$, $x-z$, and $y-z$ plane. The spiraling of the particles around the wing tip is clearly seen from Fig. 12a and Fig. 12c. Figure 12b shows that the vortex lifts off the wing, the asymptotic angle reached is $\alpha=5^{\circ}$ (the angle of attack).

The three-dimensional flow field is usually cumbersome to analyze, and an effective way to analyze such a field is to project surface sections of the field onto appropriate two-dimensional planes. Three surfaces, $\eta=15, \xi=20$, and $\xi=$ 27 , were selected to study the tip vortex formation. These surfaces intersect the surface of the wing, as shown in Fig. 10; that is, $\eta=15$ is a surface-cut of the flow field that will show the flow pattern in a section more or less parallel to the 
streamwise direction; the other two surface-cuts show the flow pattern in sections approximately normal to the streamwise direction. Figure 13 shows the projection of the velocity vector at $\eta=15$ on an $x-z$ plane. The flow-separation point and reattachment point are indicated by the arrows. The particle paths on this surface are shown in Fig. 14. Since the flow is three-dimensional, a recirculation zone is not necessary to indicate reattachment. The thickness of the separated layer is about $0.075 c$ and is of the same order as the thickness of the boundary layer at the root of the wing; that is, the separation zone is fairly thin. Figures 15 and 16 show the projection on a $y-z$ plane of the velocity vectors computed on the surfaces $\xi=20$ and $\xi=27$. The presence of a recirculation zone is more pronounced in these surface-cuts. Figure 17 shows the streamlines of the velocity vectors of Fig. 15. The core of the recirculation zone is clearly distinguishable in this figure. If this recirculation zone is taken to be the root of the tip vortex, we can conclude that the tip vortex is attached to the wing in this plane. The combination of the observations from the vector plots (Figs. 13 and 15) and the three-dimensional particle paths (Fig. 11) indicates that the vortex line on the wing surface away from the tip is almost parallel to the leading edge. Figure 18 shows the streamlines of the velocity vectors of Fig. 16. At this location, the core of the vortex is much smaller, and the center of the vortex has shifted toward the wing tip and lifted from the wing surface. The direction of this vortex is downstream. At this Mach number, we then have the picture shown in Fig. 19; that is, a vortex line parallel to the leading edge interacting with the tip vortex.

\section{Conclusions and summary}

A computer code has been developed to solve the three-dimensional, compressible thin-layer equations in generalized coordinates. The code can accommodate up to 200,000 grid points. A grid-generation system that is modular has also been developed that can be used to generate one-dimensional grid stretching, twodimensional body-fitted grids, and three-dimensional body-fitted grids. This system was used to compute the flow field around a low-aspect-ratio wing at $M=0.82$. At this Mach number, an oblique shock is observed on the upper surface of the wing. Also, a tip vortex forms and lifts off the wing. The formation of the tip vortex was analyzed. We found that the flow around the leading edge contributes to the vortex formation around the tip, and that the circulation generated in the boundary layer will also contribute to the formation of the tip vortex.

\section{Acknow ledgments}

This work was carried out under grant NAG 2-029 sponsored by NASA Ames Research Center. Partial support was also provided by Prof. J. Steger, Stanford University. The author is indebted to P. Buning of NASA Ames Research Center 
for his three-dimensional graphics package that was used to generate the figures in this paper. Special thanks go to T. Pulliam and P. Moin, NASA Ames Research Center, for numerous helpful discussions during the course of this work.

\section{References}

${ }^{1}$ Prandtl, L., "Applications of Modern Hydrodynamics to Aeronautics," NASA RP-1050, R. T. Jones ed., 1979.

${ }^{2}$ Subramanian, N. R., Holst, T. L., and Thomas, S. D., "Recent Applications of the Transonic Wing Analysis Computer Code TWING," NASA TM-84283, 1982.

${ }^{3}$ Greene, G. C., "Wake Vortex Alleviation," AIAA Paper 81-0798, Atlantic City, N. J., 1981.

${ }^{4}$ Baldwin, B. S. and Lomax, H., "Thin Layer Approximation and Algebraic Model for Separated Turbulent Flow s," AIAA Paper 78-257, 1978.

${ }^{5}$ Rizzi, A. and Eriksson, L. E., "Transfinite Mesh Generation and Damped Euler Equation Algorithm for Transonic Flow around Wing-Body Configurations," AIAA Paper 81-0999, Palo Alto, Calif., 1981.

${ }^{6}$ Eriksson, L. E. and Rizzi A., "Computation of Vortex Flow around Wings Using the Euler Equations," Proceedings of the IVth GAMM Conference on Numerical Methods in Fluid Mechanics, Vieweg-Verlag, Paris, 1981.

${ }^{7}$ Rizzi, A., "Mesh Influence on Vortex Shedding in Inviscid Flow Computations," Recent Contributions to Fluid Mechanics, W. Haase ed., Springer-Verlag, Berlin, 1982.

${ }^{8}$ Mehta, U. and Lomax, H., "Reynolds Averaged Navier-Stokes Computations of Transonic Flows-The State-of-the-Art," Transonic Aerodynamics, D. Nixon ed., Progress in Astronautics and Aeronautics, Vol. 81, 1982, pp. 297-375.

${ }^{9}$ Fujii, K. and Kutler, P., "Numerical Simulation of the Leading-Edge Separation Vortex for a Wing and Strake-Wing Configuration," AIAA Paper 83-1098, Danvers, Mass., 1983.

${ }^{10}$ Hollanders, H., Lerat, A., and Peyret, R., "3-D Calculation of Transonic Viscous Flows by an Implicit Method," AIAA Paper 83-1953, Danvers, Mass., 1983.

${ }^{11}$ Dwyer, H. A., Smooke, M. D., and Kee, R. J., "Adaptive Gridding for Finite Difference Solutions to Heat and Mass Transfer Problems," Symposium on the Numerical Generation of Curvilinear Coordinate Systems and Use in the Numerical Solution of Partial Differential Equations, Nashville, Tenn., 1982. 
${ }^{12}$ Beam, R. M. and Warming, R. F., "An Implicit Finite-Difference Algorithm for Hyperbolic Systems in Conservation-Law Form," Journal of Computational Physics, Vol. 22, No. 1, 1976, pp. 87-110.

${ }^{13}$ Briley, W. R. and McDonald, H., "Solution of the Multidimensional Compressible Navier-Stokes Equations by a Generalized Implicit Method," Journal of Computational Physics, Vol. 24, No. 4, 1977, pp. 372-397.

${ }^{14}$ Pulliam, T. H. and Steger, J. L., "Implicit Finite-Difference Simulations of Three-Dimensional Compressible Flow," AIAA Journal, Vol. 18, No. 2, 1980, pp. 159-167.

${ }^{15}$ Mastin, C. W. and Thompson, J. F., "Transformation of Three-Dimensional Body-Fitted Curvilinear Coordinate Systems for Fluid Dynamics Calculations," Numer. Math., Vol. 29, No. 4, 1978, pp. 397-407.

${ }^{16}$ Thomas, P. D., "Composite Three Dimensional Grids Generated by Elliptic Systems," AIAA Journal, Vol. 20, 1982, p. 1195.

${ }^{17}$ Thomas, P. D. and Middlecoff, J. F., "Direct Control of the Grid Distribution in Meshes Generated by Elliptic Equations," AIAA Journal, Vol. 18, 1980, pp. 652-656.

${ }^{18}$ Hinson, B. L. and Burdges, K. P., "Acquisition and Application of Transonic Wing and Far-Field Test Data For Three-Dimensional Computational Method Evaluation," Report No. AFOSR-TR-80-0421, Lockheed-Georgia Co., Marietta, Ga., 1980 .

${ }^{18}$ Holst, T. L., Subramanian, N. R., and Thomas, S. D., "The Efficient Solution of Transonic Wing Flow Fields," 2nd Symposium on Numerical and Physical Aspects of Aerodynamic Flows, Long Beach, Calif., 1982.

${ }^{20}$ Douglas, J. and Gunn, J. E., "A General Formulation of Alternating Direction Methods," Numerische Math., Vol. 6, 1964, p. 428.

${ }^{21}$ Chapman, D. R., "Computational Aerodynamics Development and Outlook," AIAA Journal, Vol. 17, No. 12, 1979, pp. 1293-1313. 

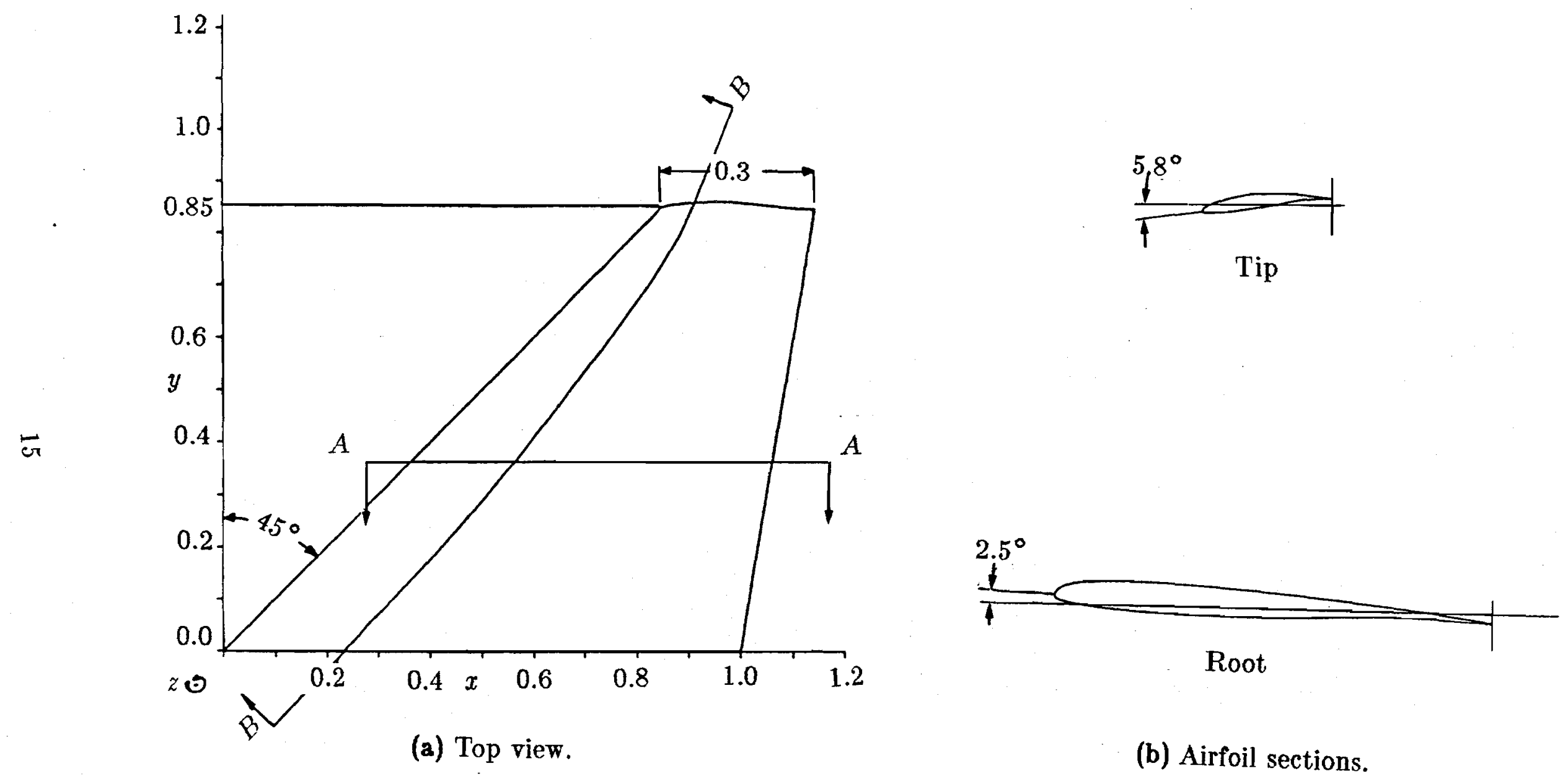

Fig. 1 Low-aspect ratio wing geometry. 


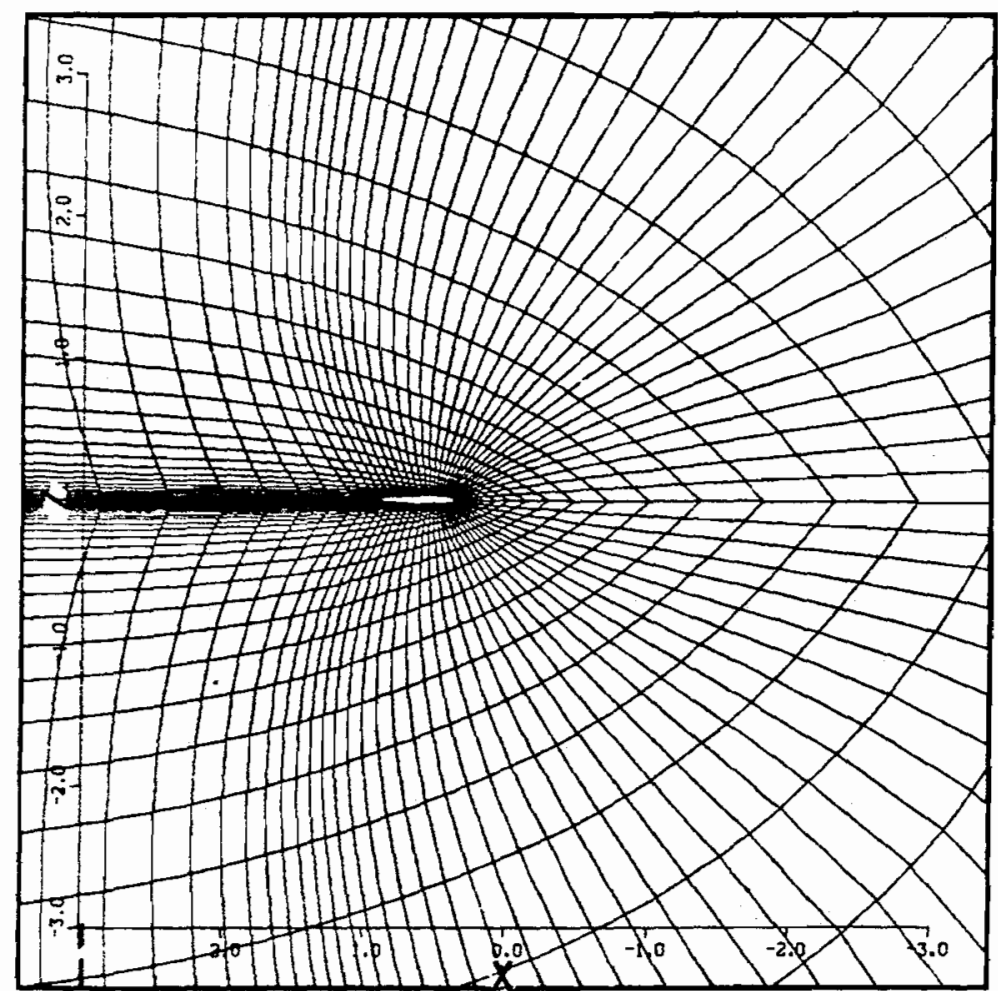

(a) Section A-A.

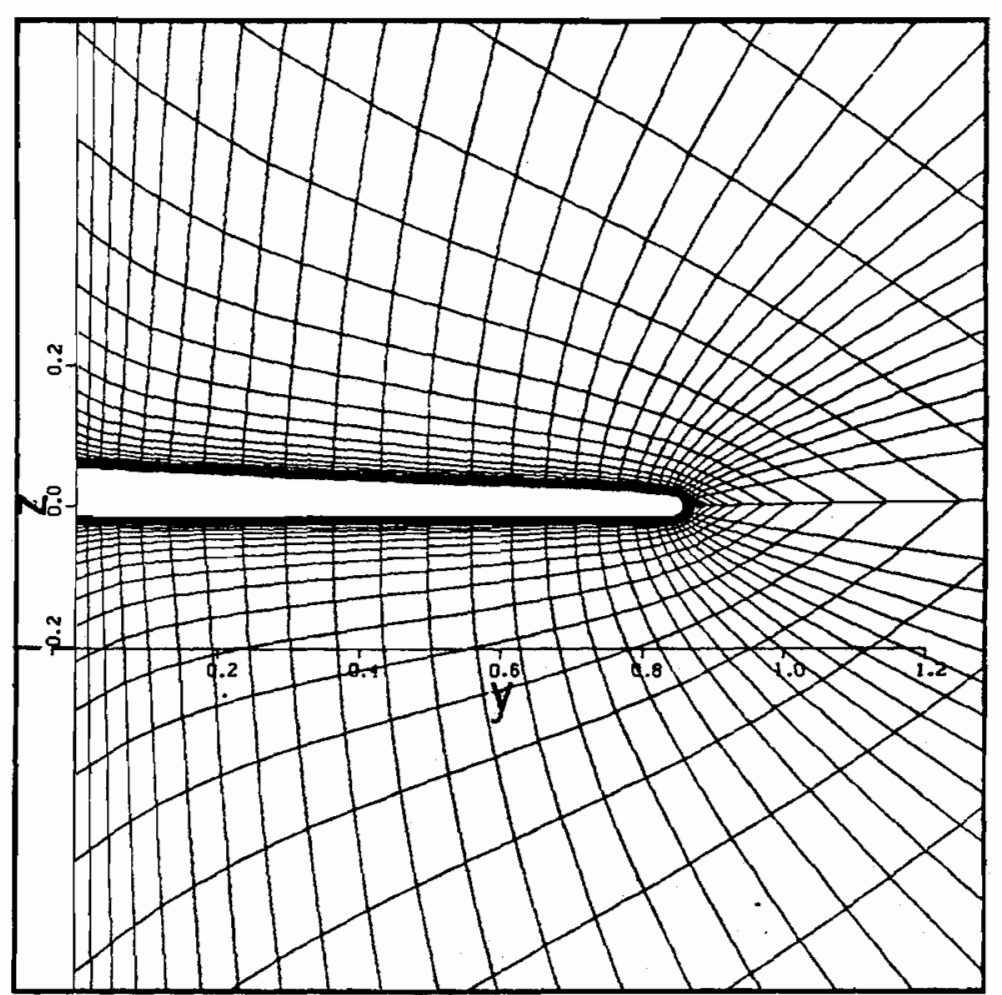

(b) Section B-B.

Fig. 2 Grid distribution. 


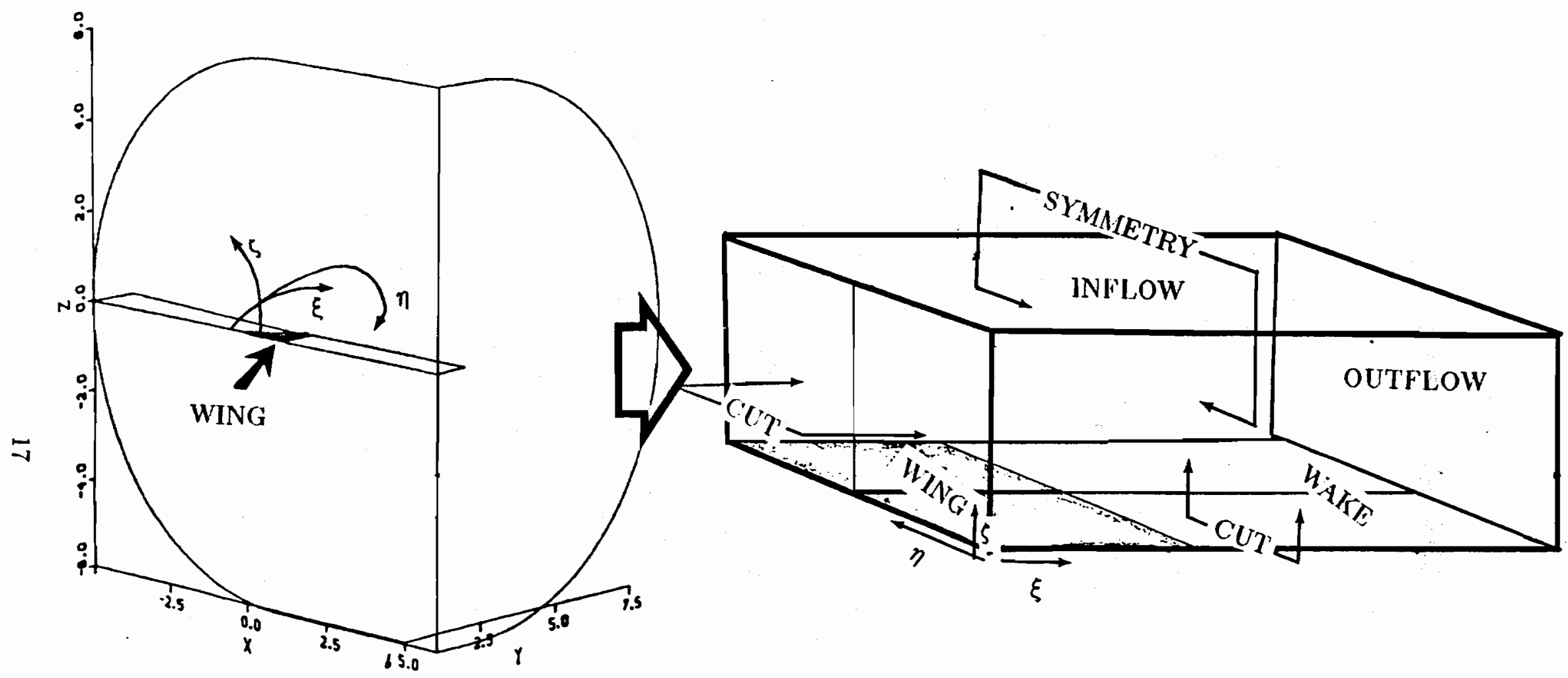

Fig. 3 Grid wrap. 


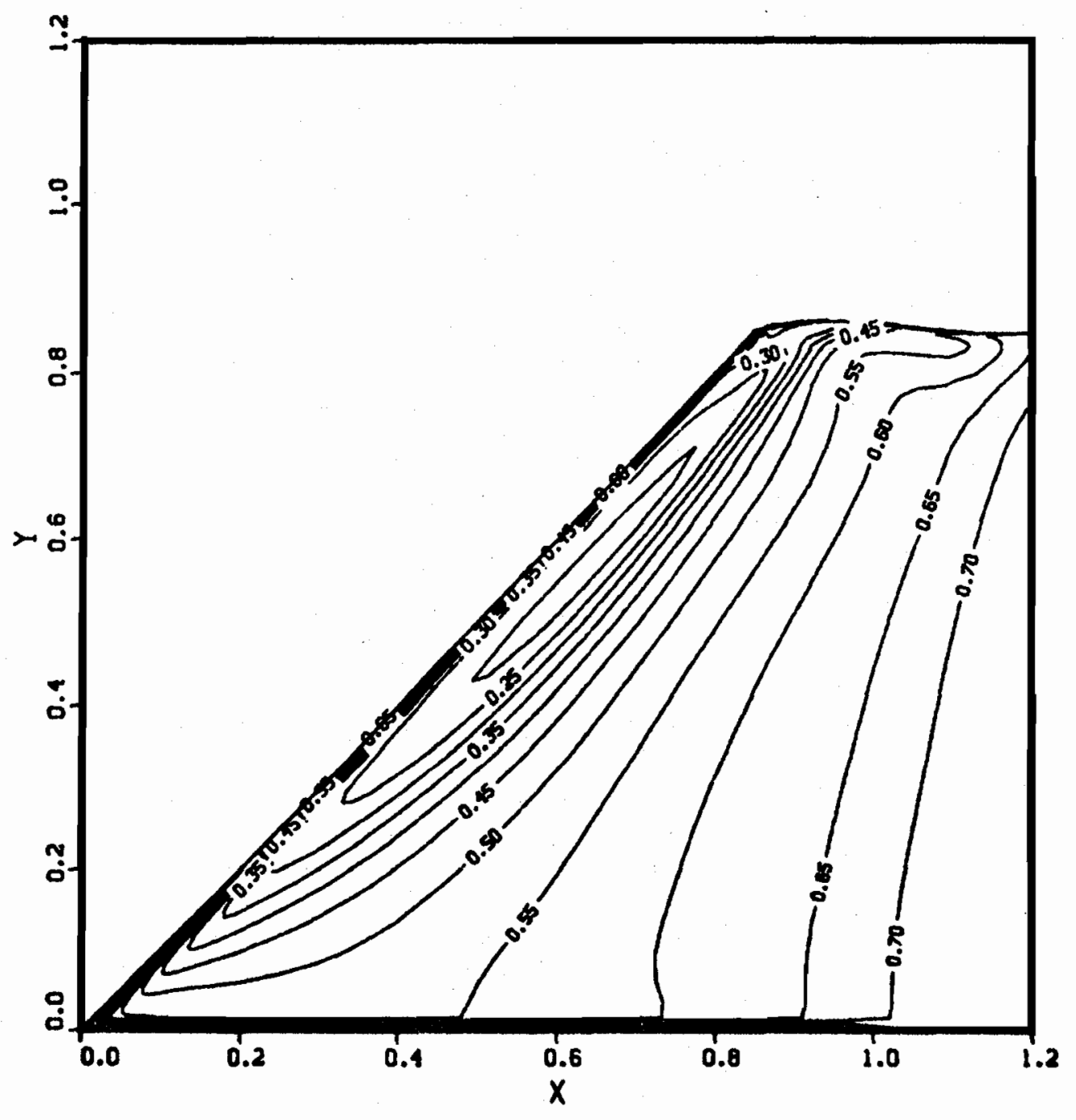

Fig. 4 Isobar lines on the wing surface. 


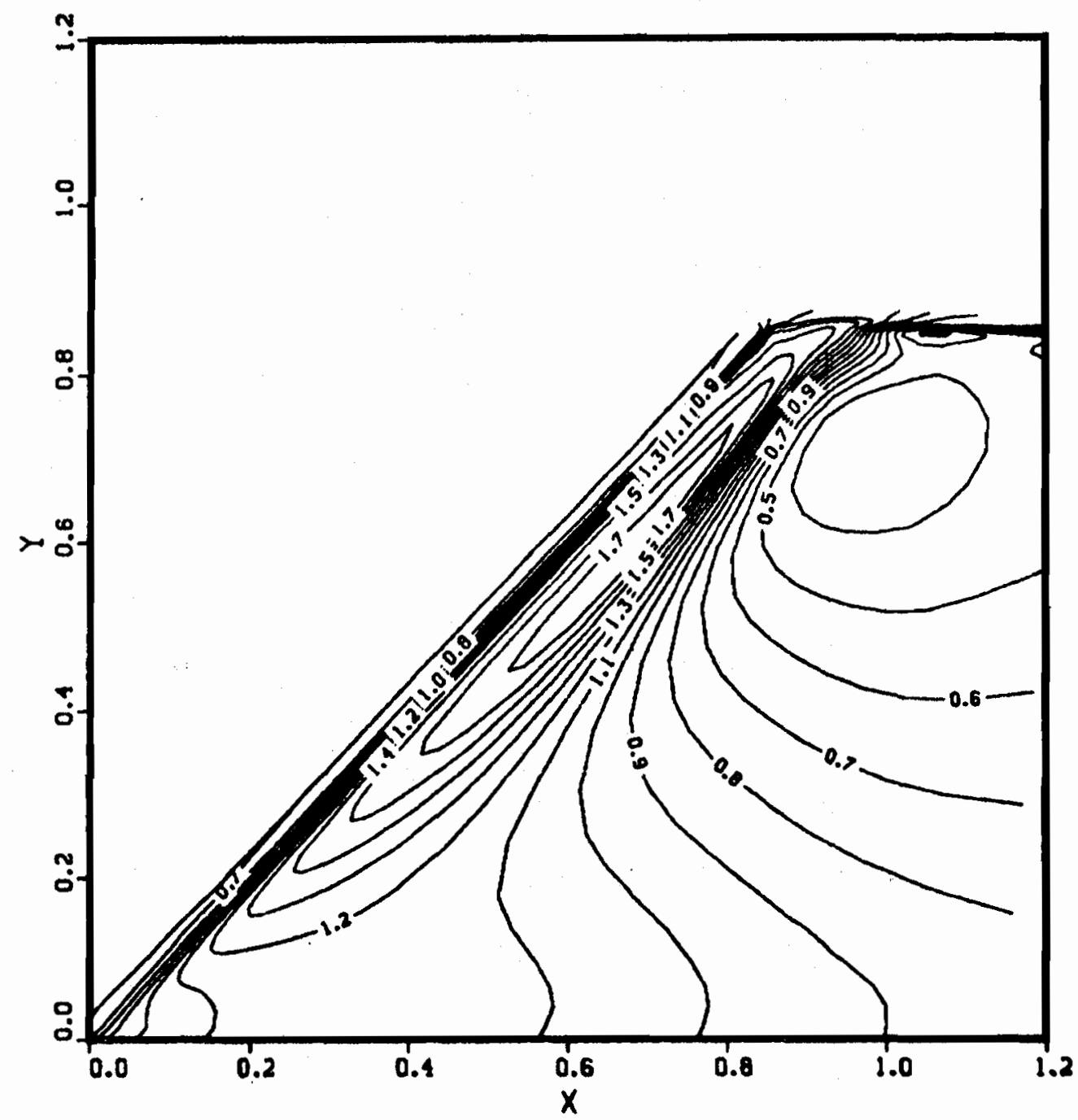

Fig. 5 Mach contours on a surface parallel to the wing surface. 


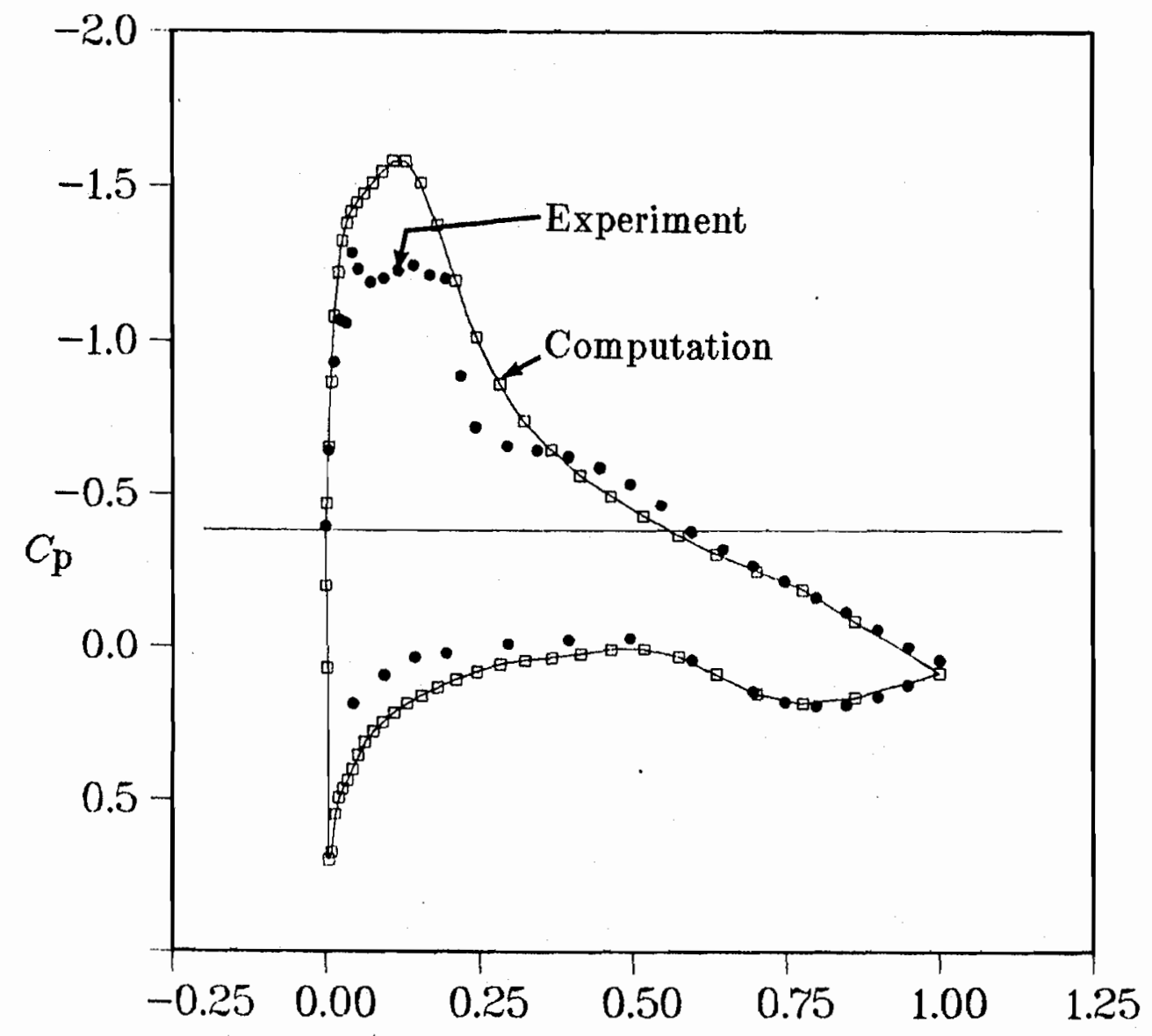

Distance from the leading edge normalized on cord length

Fig. $6 C_{\mathrm{p}}$ profiles at $y=0.6 c$. Comparison with experimental data . 


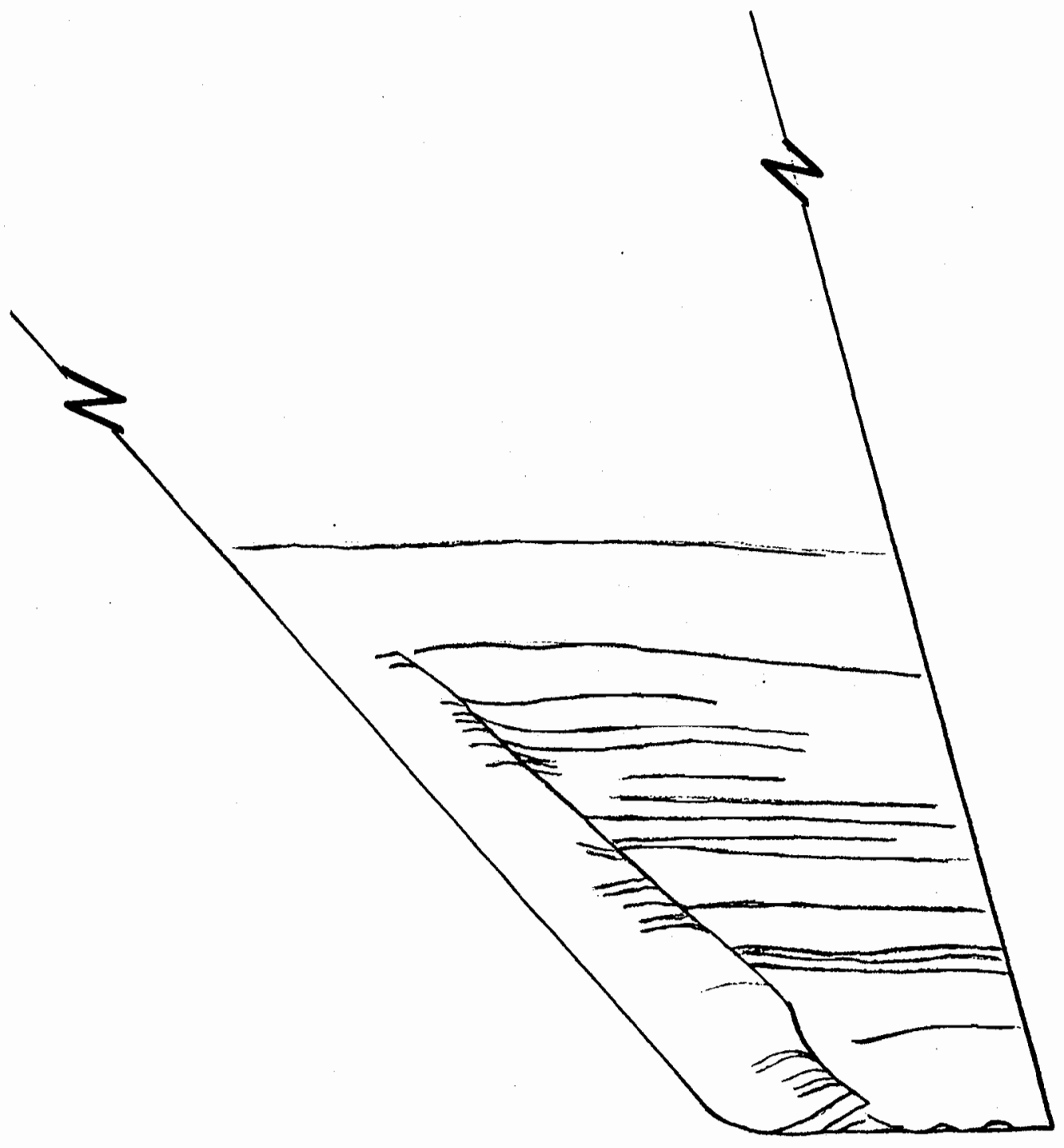

Fig. 7 Sketch of oil pattern from a photograph of the experiment of Kcener . 


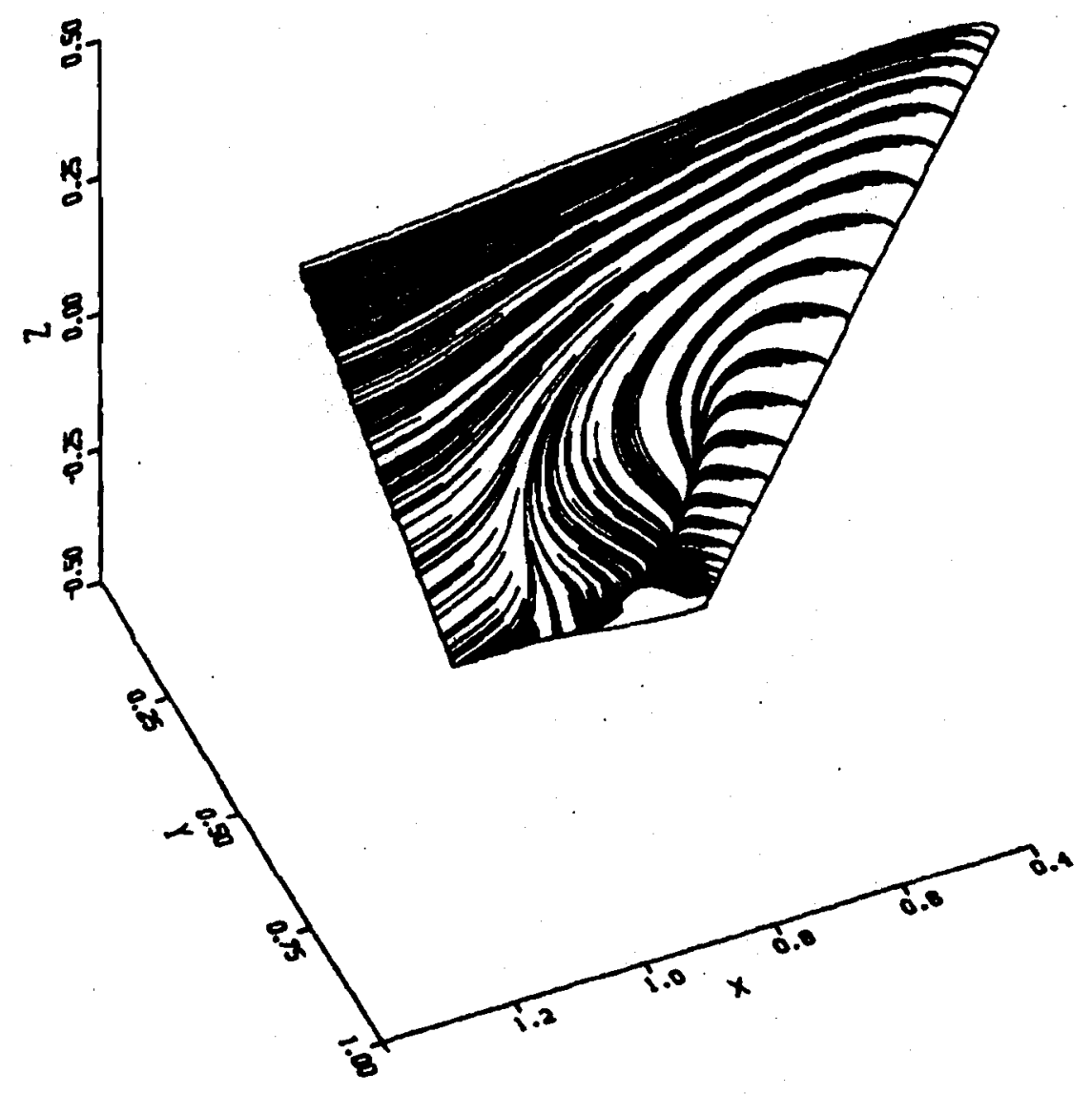

Fig. 8 Particle paths on $\zeta=2$ surface. 


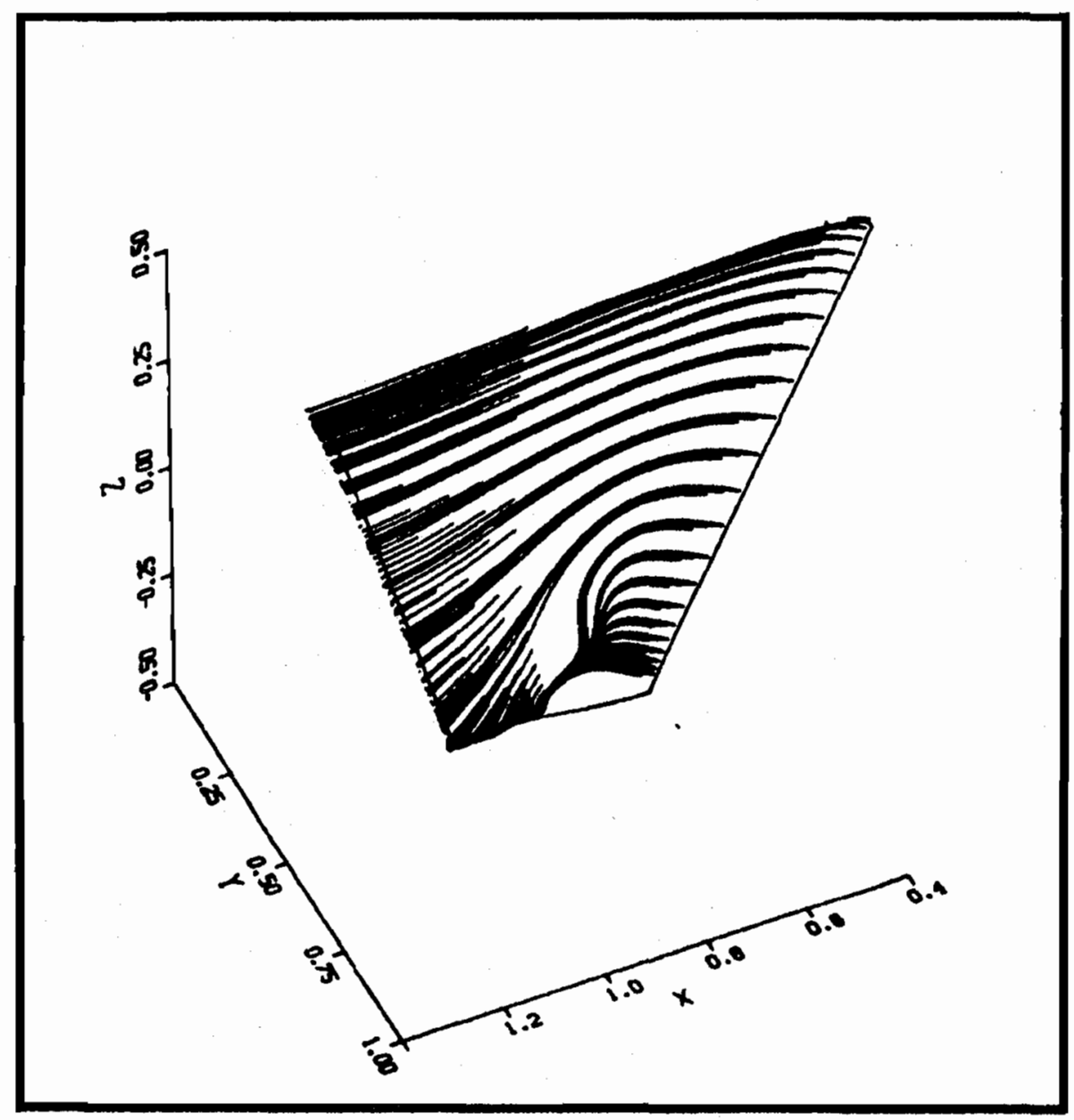

Fig. 9 Particle paths on $\zeta=8$ surface. 


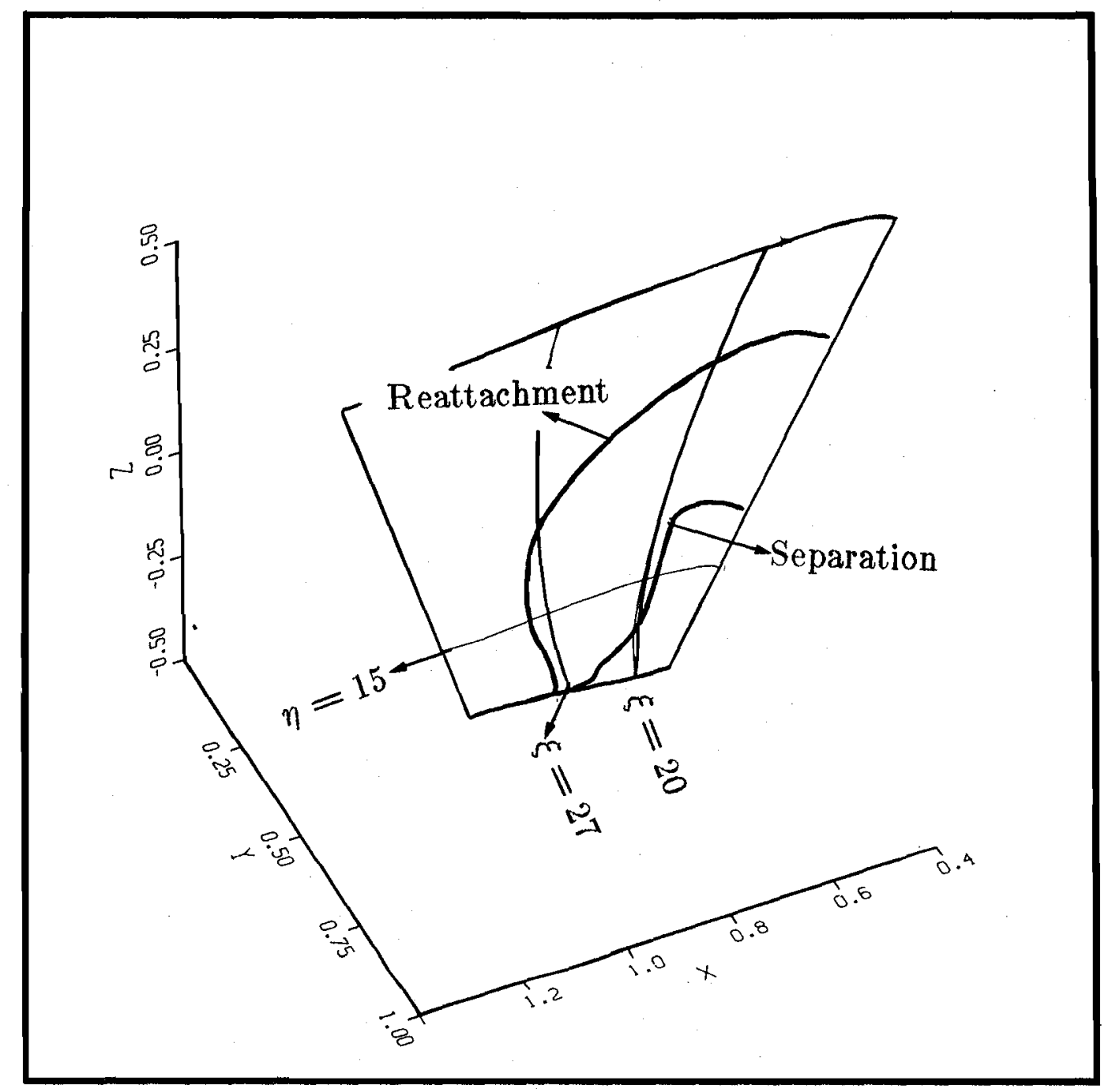

Fig. 10 Flow separation and reattachment lines. 


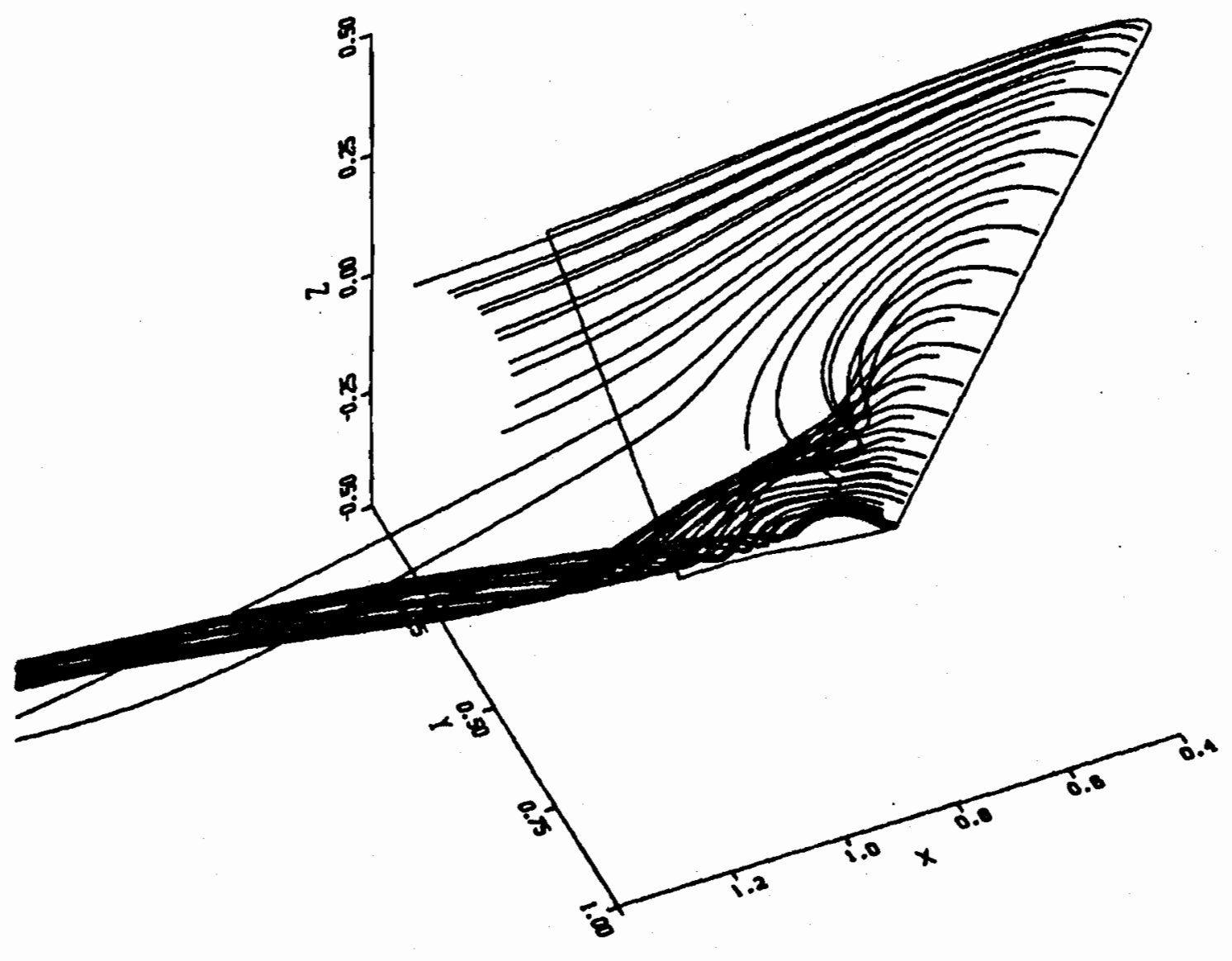

Fig. 11 Three-dimensional particle paths. 


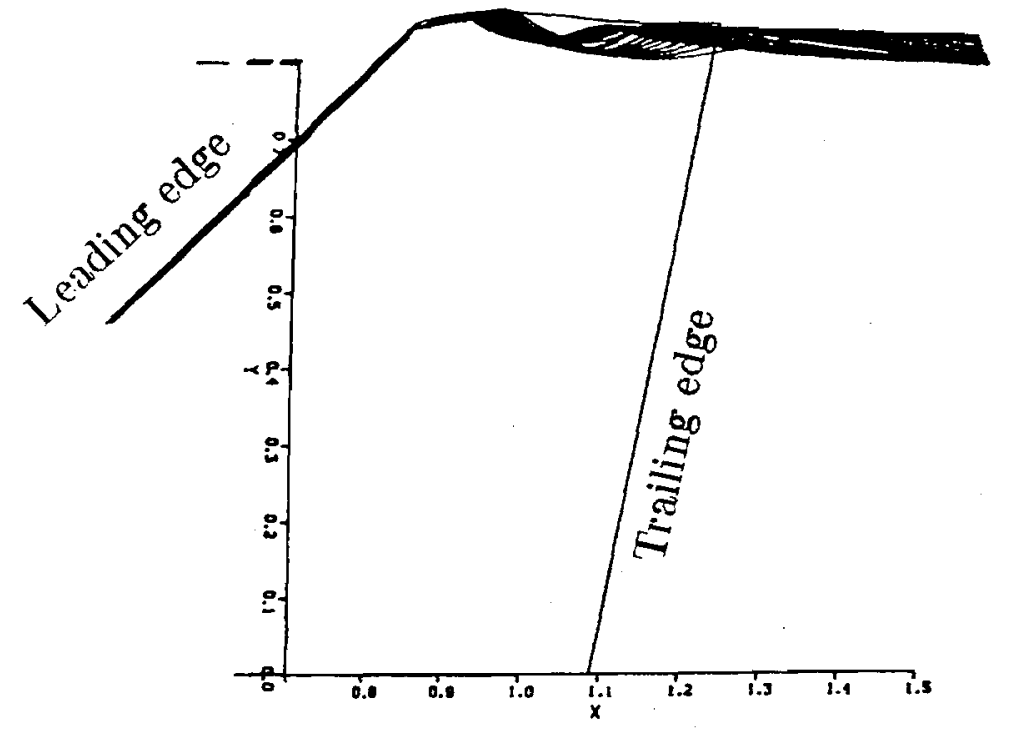

(a) $x-y$ projection.

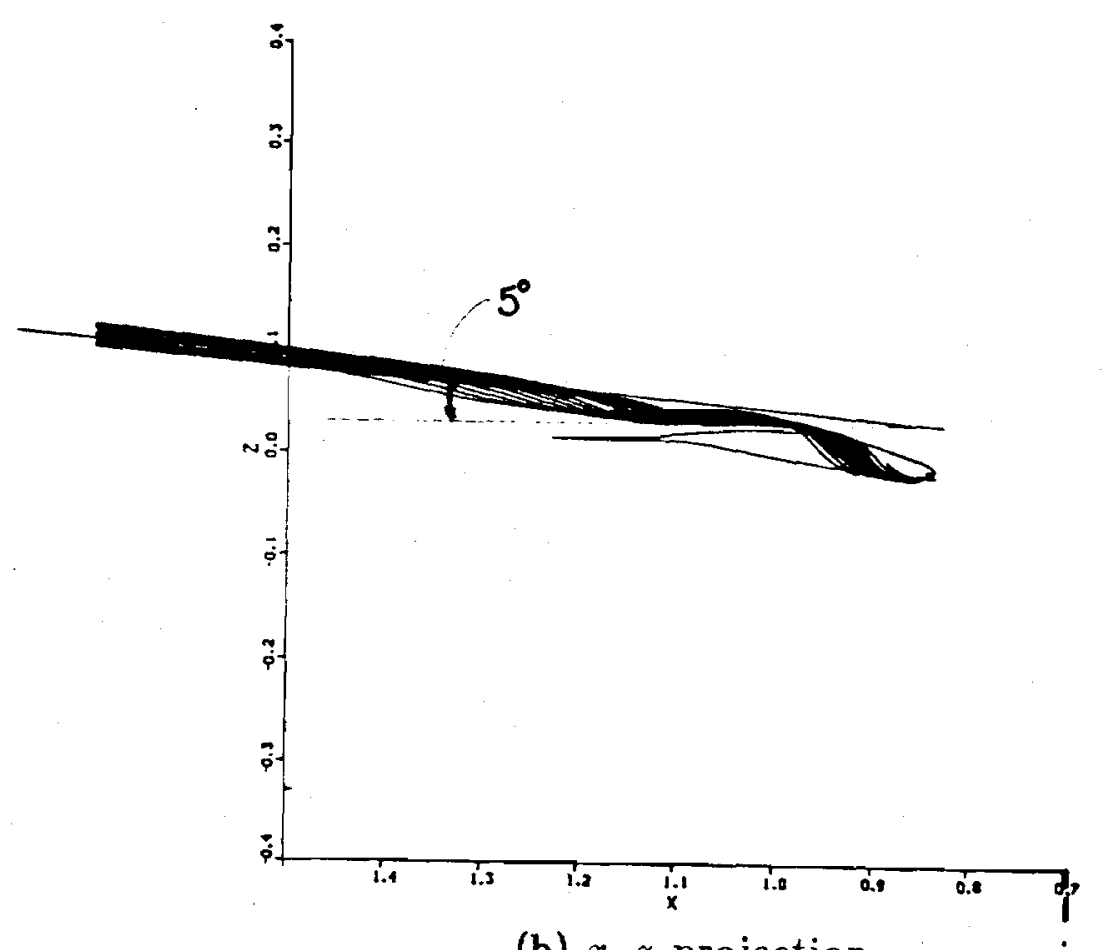

(b) $x-z$ projection.

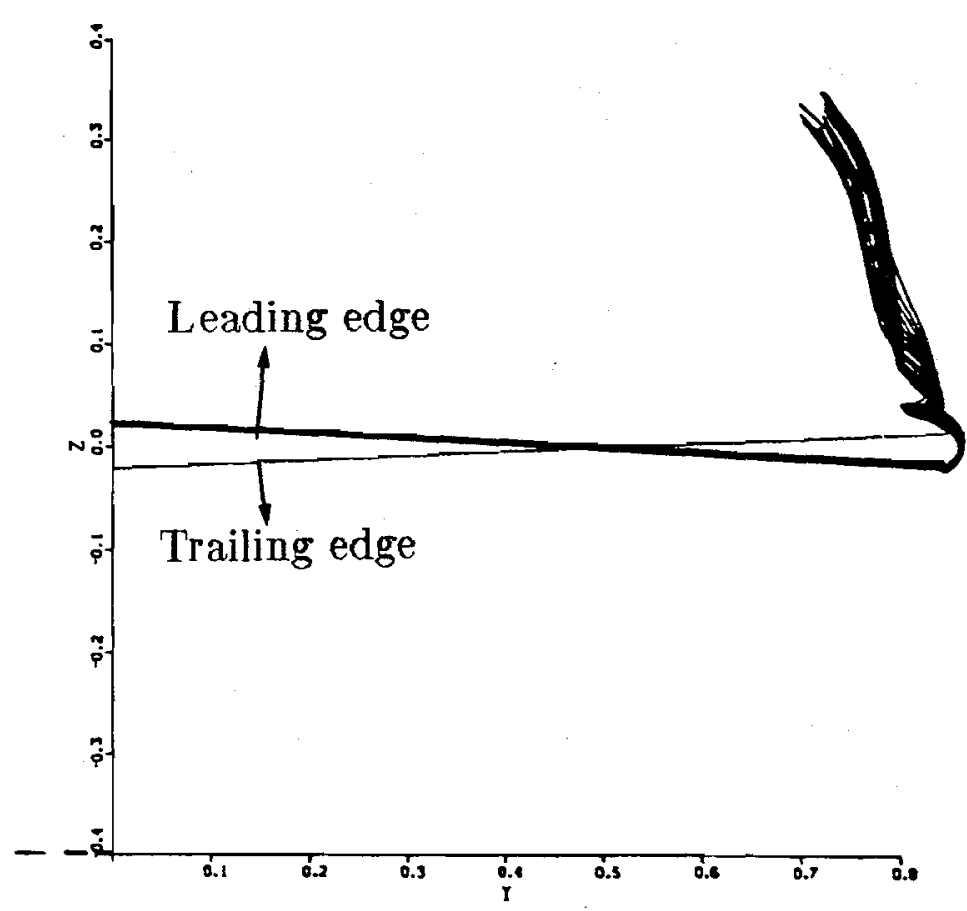

(c) $y-z$ projection

Fig. 12 The tip vortex. 


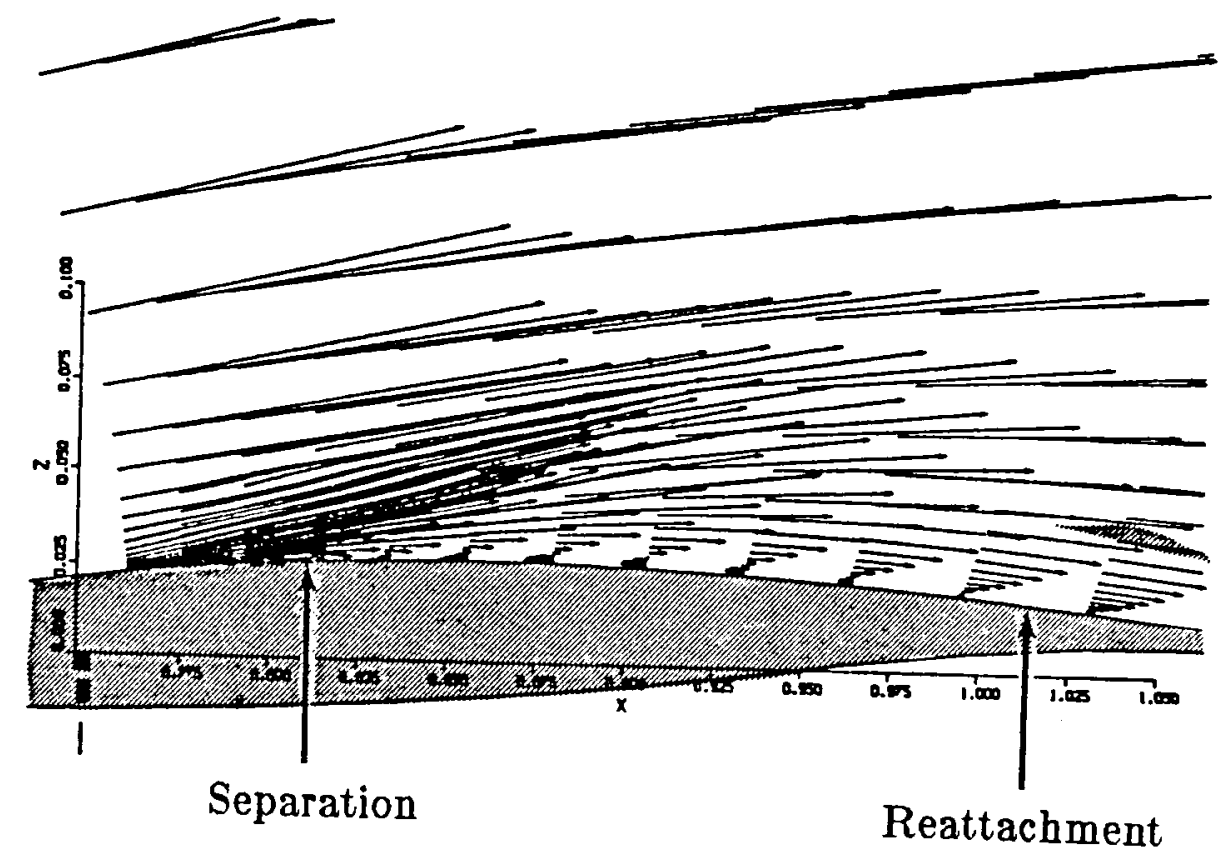

Fig. 13 Velocity vectors projection on an $x-z$ plane. (surface $\eta=15$ ). 


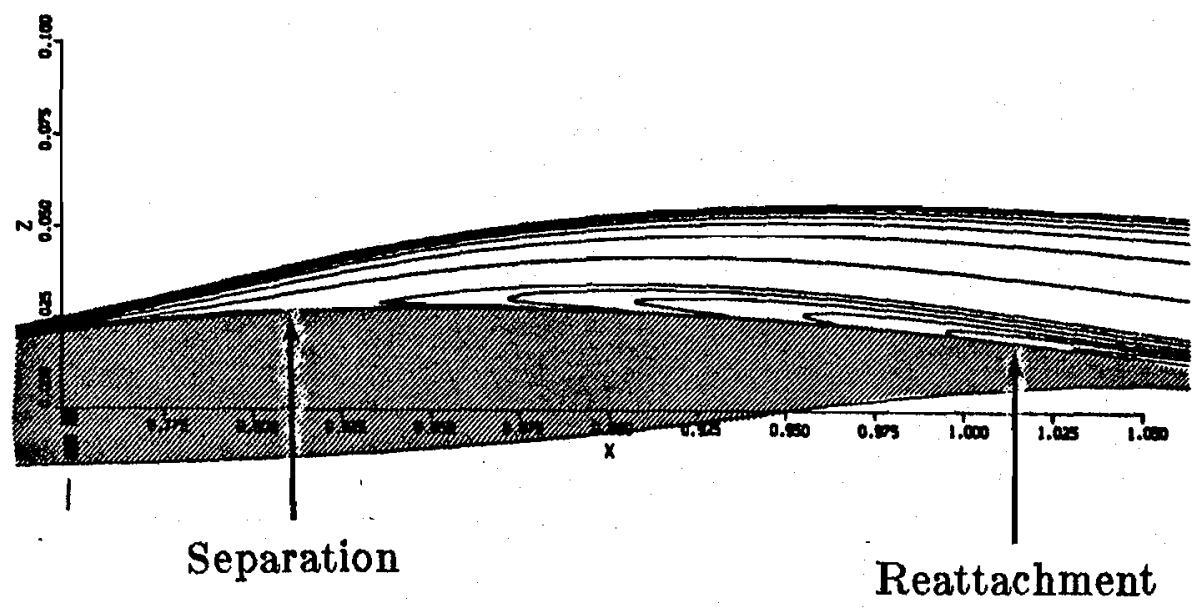

Fig. 14 Particle paths on $\eta=15$ surface. 


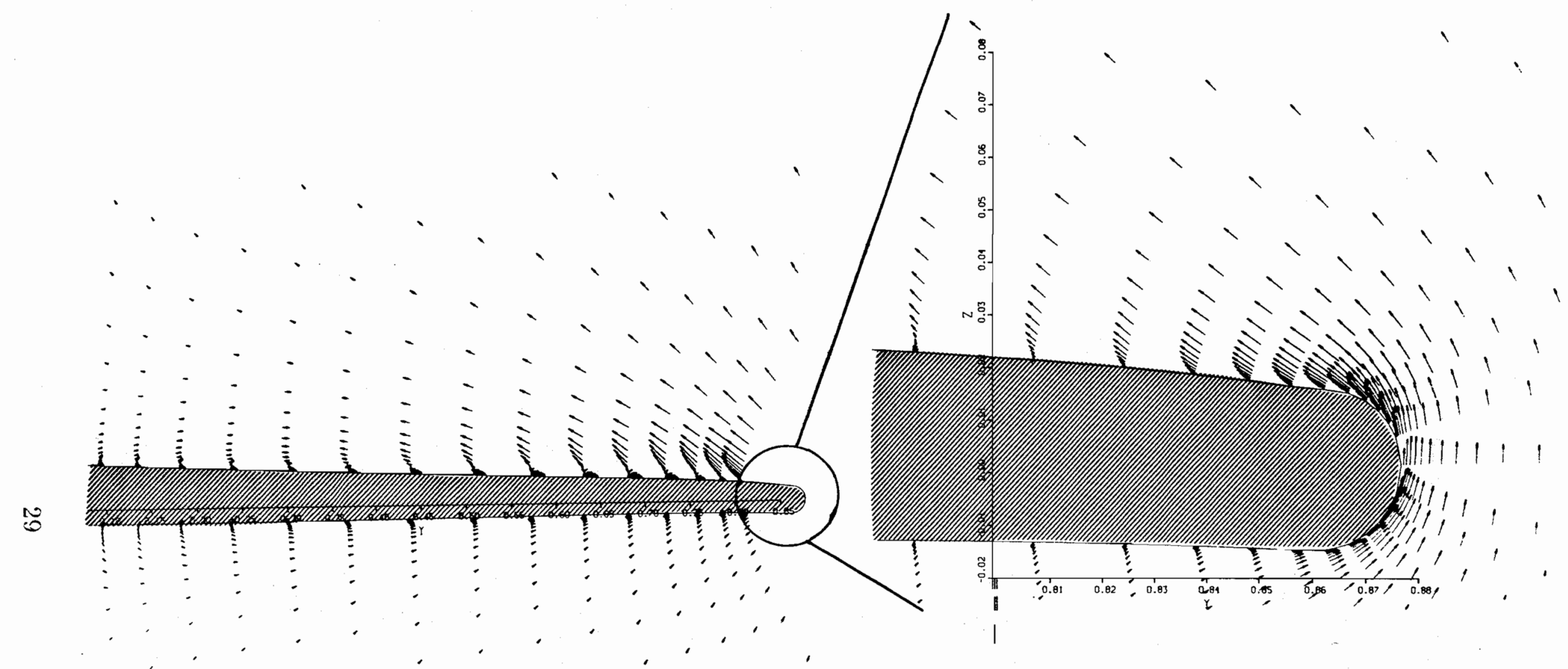

Fig. 15 Velocity vectors projection on a $y-z$ plane. (surface $\xi=20$ ). 


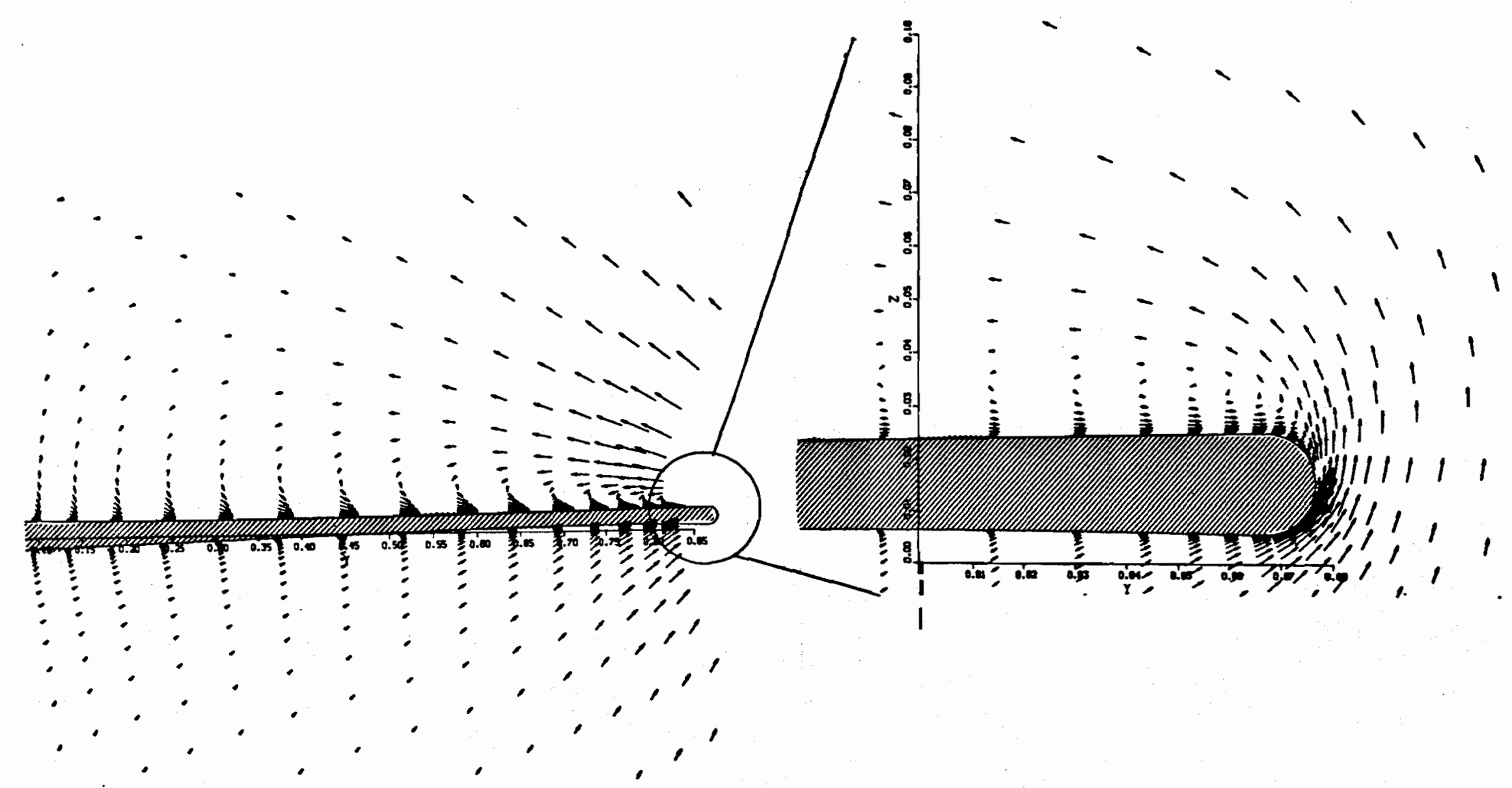

Fig. 16 Velocity vectors projection on a $y-z$ plane. (surface $\xi=27$ ) 


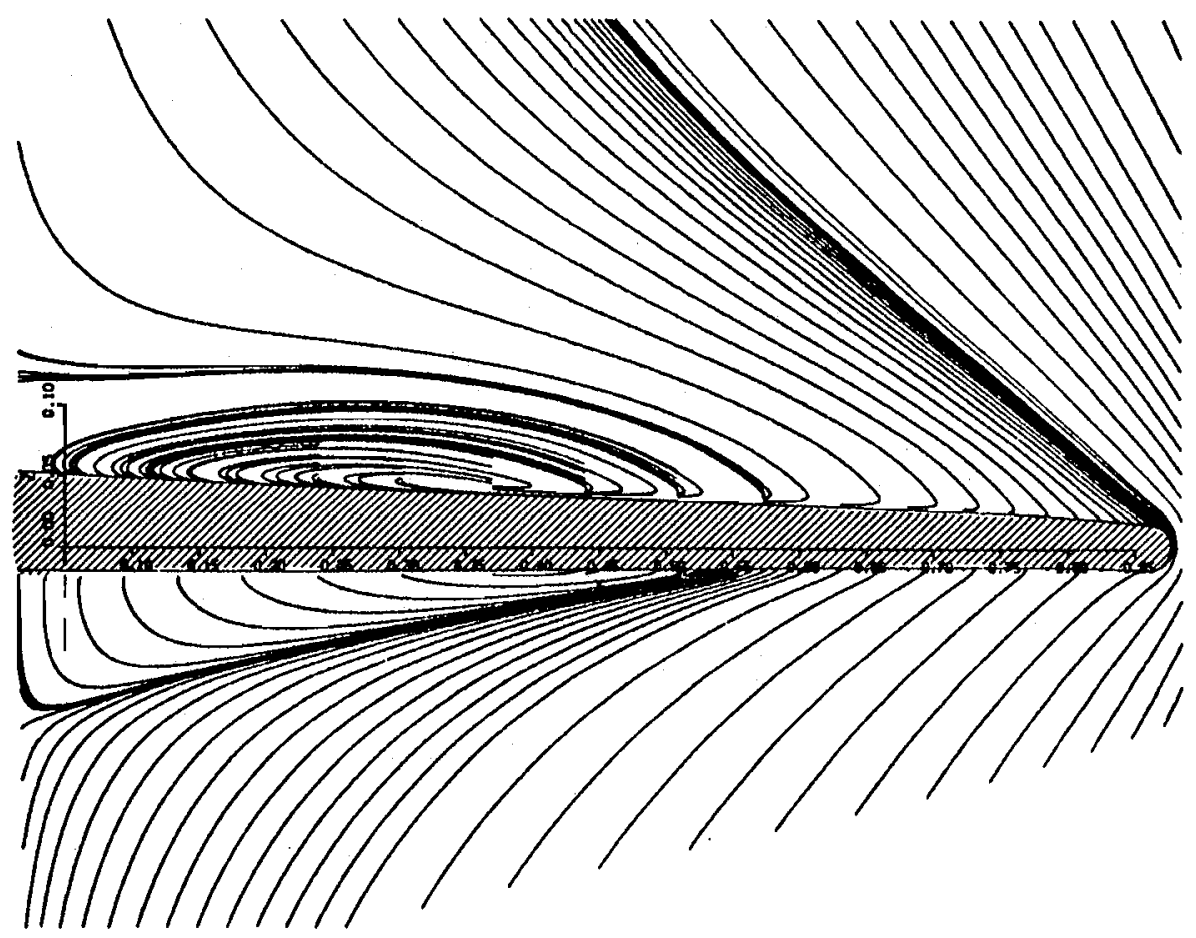

Fig. 17 Particle paths in a $y-z$ plane. (surafce $\xi=20$ ). 


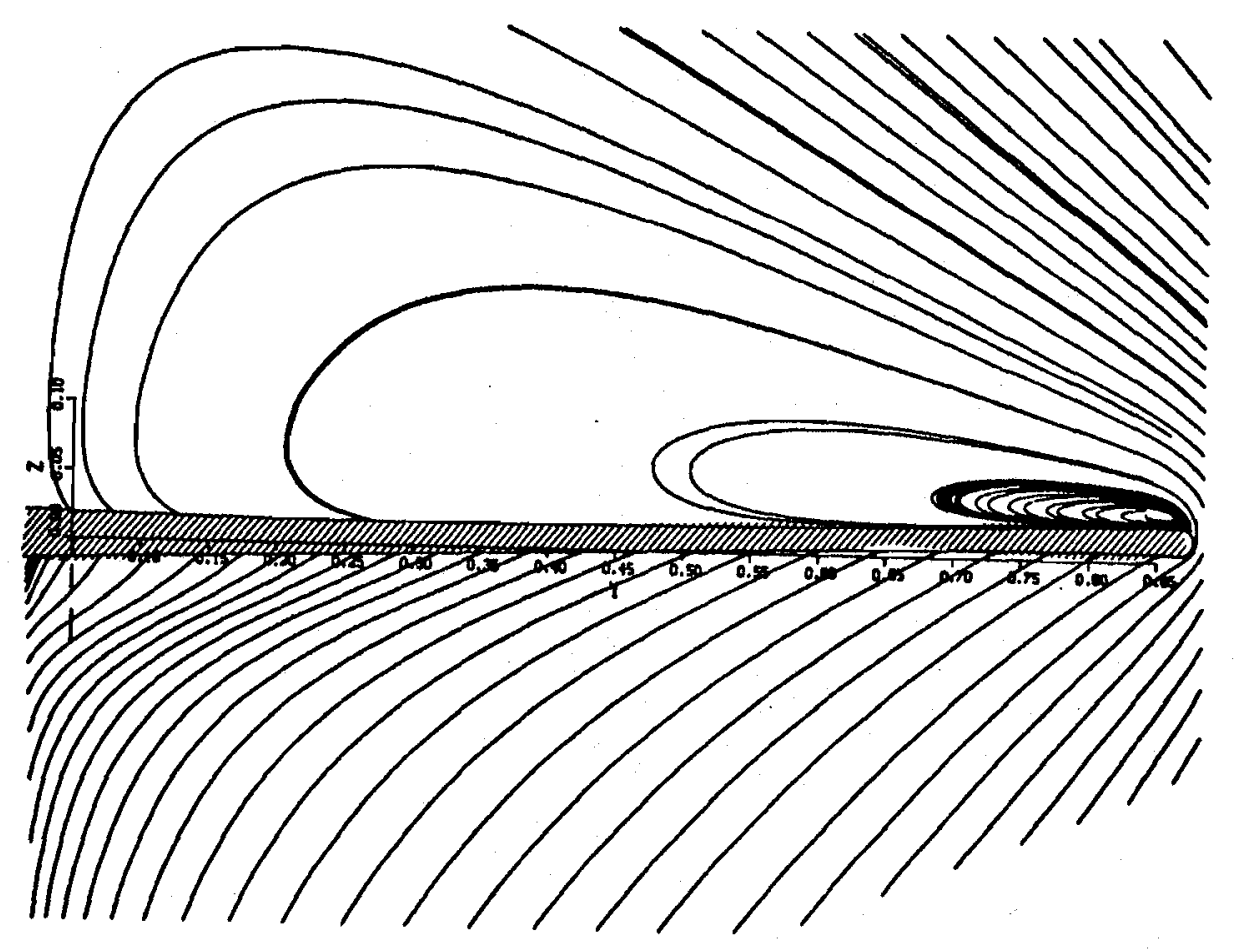

Fig. 18 Particle paths in a $y-z$ plane. (surafce $\xi=27$ ). 


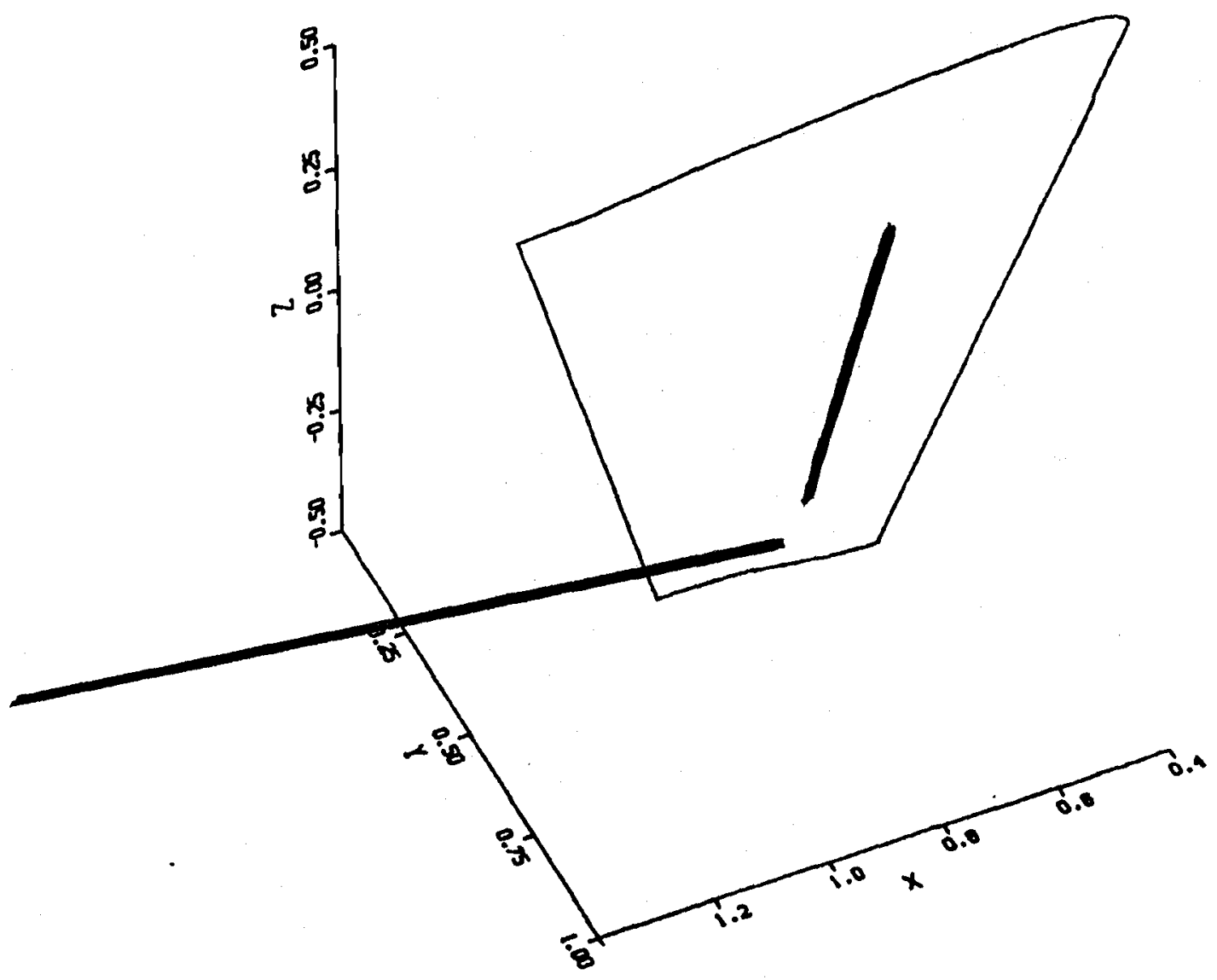

Fig. 19 Sketch of the two vortex lines interacting on the upper surface of the wing 


\begin{tabular}{|c|c|c|c|}
\hline $\begin{array}{l}\text { 1. Report No. } \\
\text { NASA TM-85932 }\end{array}$ & 2. Government Acceusion No. & \multicolumn{2}{|c|}{ 3. Recipient's Catalog No. } \\
\hline \multirow{2}{*}{\multicolumn{2}{|c|}{$\begin{array}{l}\text { 4. Title and Subtitle } \\
\text { NUMERICAL SIMULATION OF THE TIP VORTEX OFF A LOW- } \\
\text { ASPECT-RATIO WING AT TRANSONIC SPEED }\end{array}$}} & \multicolumn{2}{|l|}{$\begin{array}{l}\text { 5. Report Date } \\
\text { Apri1 } 1984\end{array}$} \\
\hline & & \multicolumn{2}{|c|}{ 6. Performing Orgenization Code } \\
\hline \multicolumn{2}{|c|}{$\begin{array}{l}\text { 7. Author(s) } \\
\text { N. N. Mansour (Stanford University, Stanford, } \\
\text { Calif.) }\end{array}$} & \multicolumn{2}{|c|}{$\begin{array}{l}\text { 8. Performing Organization Report No. } \\
\text { A-9693 }\end{array}$} \\
\hline \multirow{2}{*}{\multicolumn{2}{|c|}{$\begin{array}{l}\text { 9. Performing Organization Name and Address } \\
\text { Ames Research Center } \\
\text { Moffett Field, CA } 94035\end{array}$}} & \multicolumn{2}{|l|}{$\mathrm{T}-6465$} \\
\hline & & \multicolumn{2}{|c|}{ 11. Contract or Grant No. } \\
\hline & & \multirow{2}{*}{\multicolumn{2}{|c|}{ 13. Type of Report and Period Covered }} \\
\hline \multicolumn{2}{|l|}{ 12. Sponsoring Agency Name and Address } & & \\
\hline \multicolumn{2}{|c|}{$\begin{array}{l}\text { National Aeronautics and Space Administration } \\
\text { Washington, DC } 20546\end{array}$} & \multicolumn{2}{|c|}{$\begin{array}{l}\text { 14. Sponsoring Agency Code } \\
505-31-01\end{array}$} \\
\hline \multicolumn{4}{|c|}{ 15. Supplementary Notes } \\
\hline \multicolumn{4}{|c|}{$\begin{array}{l}\text { Point of Contact: Harvard Lomax, MS } 202 A-1 \text {, Ames Research Center, Moffett } \\
\text { Field, CA } 94035 \\
\text { (415) } 965-5124 \text { or FTS } 448-5124\end{array}$} \\
\hline \multicolumn{4}{|c|}{$\begin{array}{l}\text { The viscous transonic flow around a low-aspect-ratio wing has been } \\
\text { computed using an implicit, three-dimensional, "thin-layer" Navier-Stokes } \\
\text { solver. The grid around the geometry of interest is obtained numerically } \\
\text { as a solution to a Dirichlet problem for the cube. The geometry chosen for } \\
\text { this study is a low-aspect-ratio wing with large sweep, twist, taper, and } \\
\text { camber. The topology chosen to wrap the mesh around the wing with good tip } \\
\text { resolution is a C-0 type mesh. Using this grid, the flow around the wing } \\
\text { was computed for a free-stream Mach number of } 0.82 \text { at an angle of attack of } \\
5^{\circ} \text {. At this Mach number, an oblique shock forms on the upper surface of } \\
\text { the wing, and a tip vortex and three-dimensional flow separation off the } \\
\text { wing surface are observed. Particle path lines indicate that the three- } \\
\text { dimensional flow separation on the wing surface is part of the roots of } \\
\text { the tip-vortex formation. The lifting of the tip vortex before the wing } \\
\text { trailing edge is clearly observed by following the trajectory of particles } \\
\text { released around the wing tip. }\end{array}$} \\
\hline $\begin{array}{l}\text { 17. Key Words (Suggested by Author(s)) } \\
\text { Turbulence modeling; Transonic flows; } \\
\text { Low-aspect-ratio wing; 3-D viscous } \\
\text { computation; Tip vortex; Vortex } \\
\text { generation }\end{array}$ & $\begin{array}{r}\text { 18. Distribution Stat } \\
\text { Unlim: }\end{array}$ & Subject $\mathrm{Ca}$ & gory -02 \\
\hline $\begin{array}{l}\text { 19. Security Classif. lof this report) } \\
\text { Unclassified }\end{array}$ & ssified & $\begin{array}{l}\text { 21. No. of Pages } \\
36\end{array}$ & $\begin{array}{l}\text { 22. Price: } \\
\mathrm{A} 03\end{array}$ \\
\hline
\end{tabular}

- For sale by the National Technical Information Sorvice, Springfield, Virginia 22161 
